\title{
Extracellular matrix mechanical cues regulate lipid metabolism through Lipin-1 and SREBP
}

\author{
Patrizia Romani', Irene Brian', Giulia Santinon', Arianna Pocaterra', Matteo Audano², Silvia Pedretti², \\ Samuel Mathieu ${ }^{3,4}$, Mattia Forcato ${ }^{5}{ }^{5}$, Silvio Bicciato ${ }^{5}{ }^{5}$, Jean-Baptiste Manneville ${ }^{3,4}$, Nico Mitro ${ }^{2}{ }^{2}$ \\ and Sirio Dupont ${ }^{1 \star}$
}

\begin{abstract}
Extracellular matrix (ECM) mechanical cues have powerful effects on cell proliferation, differentiation and death. Here, starting from an unbiased metabolomics approach, we identify synthesis of neutral lipids as a general response to mechanical signals delivered by cell-matrix adhesions. Extracellular physical cues reverberate on the mechanical properties of the Golgi apparatus and regulate the Lipin-1 phosphatidate phosphatase. Conditions of reduced actomyosin contractility lead to inhibition of Lipin-1, accumulation of SCAP/SREBP to the Golgi apparatus and activation of SREBP transcription factors, in turn driving lipid synthesis and accumulation. This occurs independently of YAP/TAZ, mTOR and AMPK, and in parallel to feedback control by sterols. Regulation of SREBP can be observed in a stiffened diseased tissue, and contributes to the pro-survival activity of ROCK inhibitors in pluripotent stem cells. We thus identify a general mechanism centered on Lipin-1 and SREBP that links the physical cell microenvironment to a key metabolic pathway.
\end{abstract}

E. ach tissue has a specific composition of its extracellular matrix $(\mathrm{ECM})$, which is associated with distinctive physical and mechanical properties. These mechanical properties are important for tissue structure, but also control cell function in physiology and disease ${ }^{1,2}$. Cells sense the mechanical properties of the ECM through integrin receptors, and measure them by adjusting the contractility of their F-actin cytoskeleton: contractility is maximal when cells are free to spread on stiff ECM substrata, while it is progressively decreased on a soft ECM or in conditions of limited spreading ${ }^{1}$. This is sufficient to control the switch between proliferation, differentiation and death in very diverse cell types, by regulating intracellular signalling pathways such as YAP (Yes-associated protein)/TAZ (transcriptional co-activator with PDZ-binding motif, also known as WWTR1) ${ }^{3,4}$ and SRF (serum response factor) ${ }^{5,6}$. In support of this model, inhibition of key players that maintain F-actin contractility including the small GTPase RHO, ROCK (RHO kinase), MLCK (myosin light chain kinase) and non-muscle myosin (NMII) induce similar responses to a soft $\mathrm{ECM}^{1}$. Yet, which other general aspects of cell biology are regulated by mechanical cues, and through which mechanism(s), remain largely unexplored. This is especially true in the case of metabolism, a fundamental engine that is constantly remodelled to match the energetic and biosynthetic requirements of the cell, whose connections to mechanical cues are only starting to emerge ${ }^{7,8}$.

\section{Results}

Actomyosin regulates lipid metabolism. To test in an unbiased manner the possibility that actomyosin contractility regulates metabolism we used global metabolomics to compare cells in conditions of high contractility (plated on plastics) with cells in conditions of low contractility, by inhibiting ROCK and MLCK. Analysis of steady-state levels of multiple metabolites indicated clear differences between controls and treated cells (Fig. 1a and Supplementary
Fig. 1a-e). The most significant and quantitative changes, which were maintained or increased between 6 and $24 \mathrm{~h}$, were the accumulation of several lipid molecules (Supplementary Fig. 1e and ref. ${ }^{9}$ ).

We validated this initial observation with a targeted lipidomic analysis at $24 \mathrm{~h}$, which confirmed the accumulation of triglycerides, diacylglycerols, lyso-phospholipids and ceramides, while many other lipid species remained unchanged overall (Fig. 1b-d, Supplementary Fig. (f-h and ref. ${ }^{9}$ ). This was accompanied by increased free and total fatty acids and cholesterol in cell extracts (Fig. 1e,f), and by a corresponding accumulation of cholesterol and neutral lipids in fixed cells (by Filipin and ORO stains, respectively; Fig. $1 \mathrm{~g}$ and Supplementary Fig. 1i-k). Such accumulation was visible after $6 \mathrm{~h}$ of treatment and was sustained up to $48 \mathrm{~h}$ (Supplementary Fig. 11). A similar effect was observed by replacing Y27632 with Fasudil, an alternative ROCK inhibitor, or by using Y27632 or ML7 inhibitors alone (Fig. 1g). Accumulation of lipids was mainly due to increased synthesis, because it was impaired in cells where rate-limiting enzymes of cholesterol and fatty-acid synthesis were inhibited by cerivastatin or 5-(tetradecyloxy)-2-furoic acid (TOFA) (Fig. 1g). Finally, lipid accumulation was a general response to ECM mechanical cues and actomyosin contractility because expression of the RHO inhibitor C3, treatment of cells with the NMII smallmolecule inhibitor blebbistatin, or seeding cells on soft versus stiff fibronectin-coated polyacrylamide hydrogels induced a coherent accumulation of cholesterol and lipid droplets (Fig. 1h,i). We then extended these findings in multiple cell types (including primary, immortalized and transformed cells) of both epithelial and connective tissue origin, indicating that lipid accumulation is a widespread response to conditions of reduced actomyosin contractility (Fig. 1j,k and Supplementary Fig. $1 \mathrm{~m}, \mathrm{n}$ ).

ECM mechanical cues regulate a genetic programme for lipid synthesis. Thinking of potential mechanisms underlying this

'Department of Molecular Medicine (DMM), University of Padua, Padua, Italy. ${ }^{2}$ Department of Pharmacological and Biomolecular Sciences (DiSFeB), University of Milan, Milan, Italy. ${ }^{3}$ Institut Curie, PSL Research University, CNRS, UMR 144, Paris, France. ${ }^{4}$ Sorbonne Université, UPMC University Paris 06 , CNRS, UMR 144, Paris, France. ${ }^{5}$ Department of Life Sciences, University of Modena and Reggio Emilia, Modena, Italy. *e-mail: sirio.dupont@unipd.it 


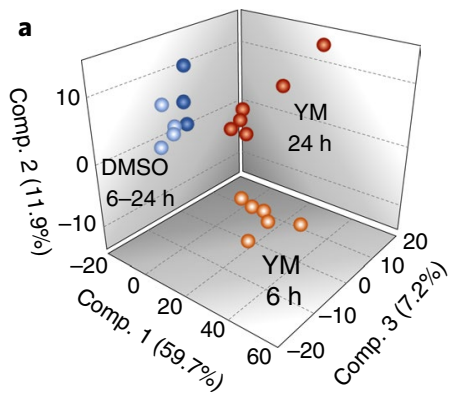

e

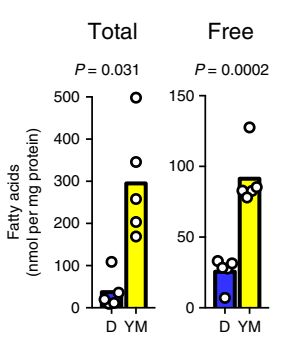
f
Tota

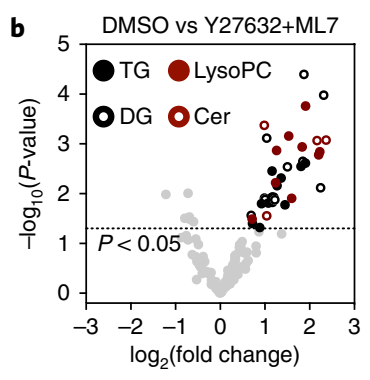

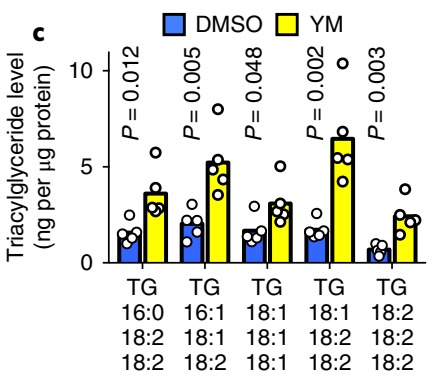

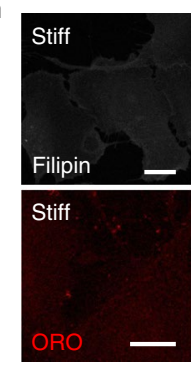

\section{Fre}
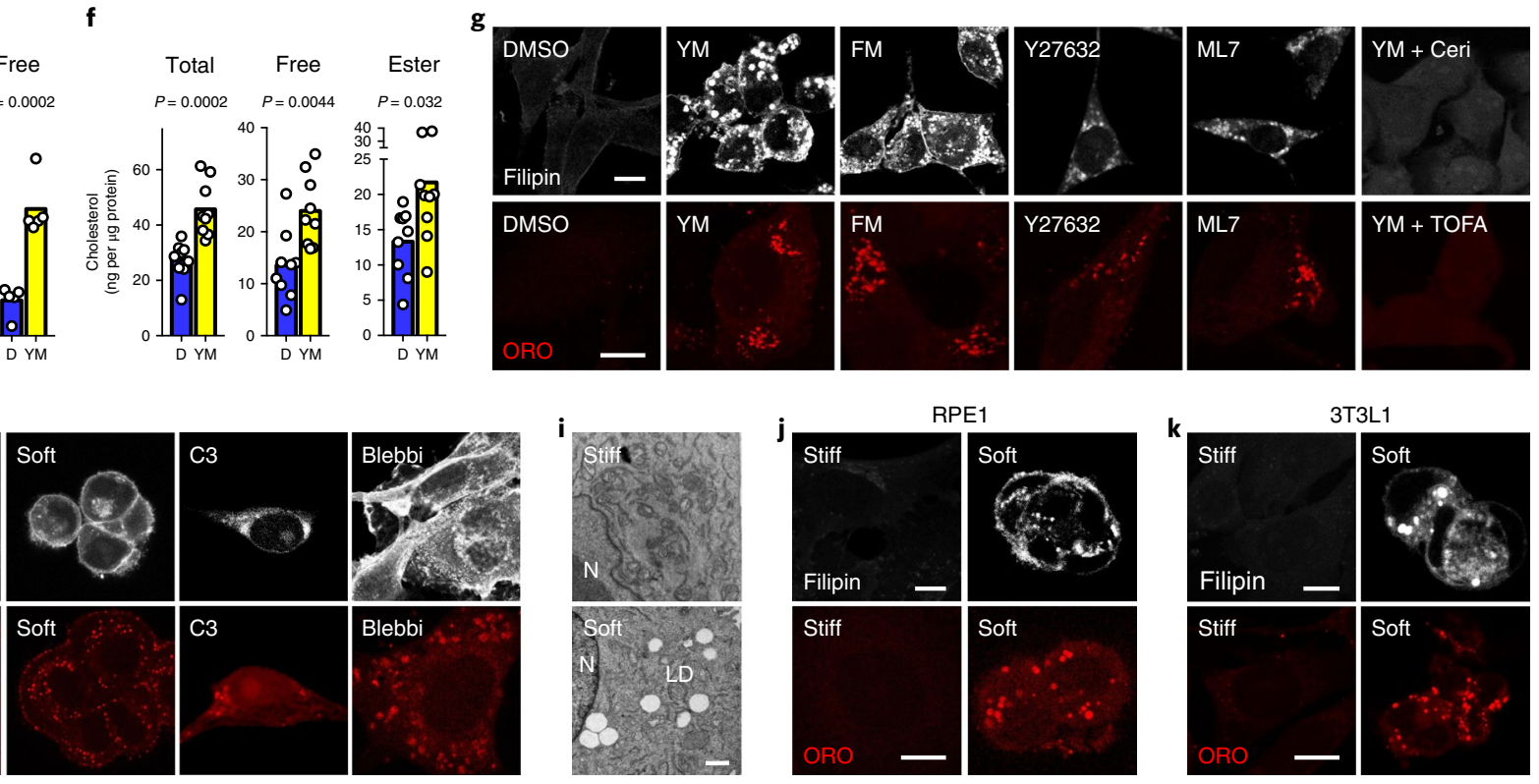

RPE1
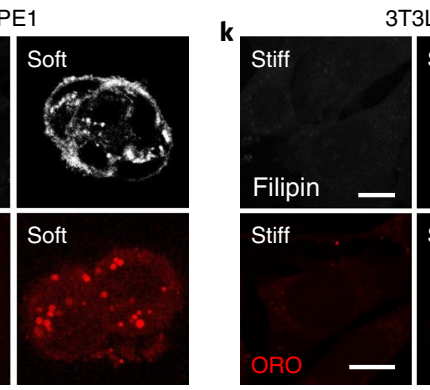

3T3L1

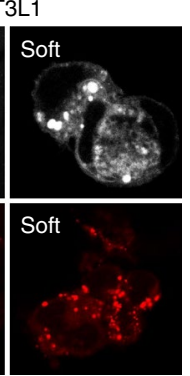

Fig. 1 Actomyosin contractility and ECM mechanical cues regulate lipid synthesis. a, Principal component analysis of metabolites altered by global metabolomics in MCF10ATk1 human mammary epithelial cells treated for 6 or $24 \mathrm{~h}$ with $20 \mu \mathrm{M}$ Y27632 ROCK inhibitor and $20 \mu \mathrm{M} \mathrm{ML7} \mathrm{MLCK} \mathrm{inhibitor}$ to inhibit actomyosin contractility (hereafter $Y M, n=6$ biologically independent samples), as compared to vehicle (DMSO, $n=4$ biologically independent samples). b. Volcano plot of lipid molecules altered in MCF10ATk1 cells treated for $24 \mathrm{~h}$ with YM, as measured by targeted lipidomics. $n=5$ biologically independent samples per condition. TG, triacylglycerols; DG, diacylglycerols; LysoPC, lyso-phosphatidylcholines; Cer, ceramides. c,d, TG (c) and DG (d) levels in MCF10ATk1 cells treated with YM for $24 \mathrm{~h}$, as measured by mass spectrometry. Only the five most abundant species are shown. Data in a-d are from a single experiment. e,f, Fatty acids (e, $n=5$ biologically independent samples per condition, one single experiment) and cholesterol (f, $n=9$ biologically independent samples pooled across two independent experiments per condition) in MCF10ATk1 cells treated with YM, as assayed by standard colorimetric assays. g, Filipin staining for cholesterol and Oil-Red-O staining (ORO) for neutral lipids in MCF10ATk1 cells treated with YM or FM (Fasudil+ ML7). Inhibition of cholesterol and fatty acid synthesis with $10 \mu \mathrm{M}$ cerivastatin (Ceri) or with $15 \mu \mathrm{M}$ TOFA prevents accumulation. Here, and in all figures, image acquisition settings were the same between controls and experimental samples. Scale bars, $5 \mu \mathrm{m}$. $\mathbf{h}$, Accumulation of cholesterol and neutral lipids in MCF10ATk1 cells on inhibition of RHO (C3 transferase transfection), non-muscle myosin II ( $20 \mu \mathrm{M}$ blebbistatin) or by plating cells on soft ( $E \approx 0.5 \mathrm{kPa})$ fibronectin-coated polyacrylamide hydrogels, compared to stiff $(E \approx 15 \mathrm{kPa})$ hydrogels. Scale bars, $5 \mu \mathrm{m}$. $\mathbf{i}$, Transmission electron microscope images of MCF10ATk1 cells plated on stiff or soft hydrogel, with apparent lipid droplets (LD) and part of the nucleus (N). Scale bar, $1 \mu \mathrm{m}$. j,k, Non-transformed human RPE1 (j) and primary mouse 3T3L1 (k) cells plated on stiff or soft hydrogels. Scale bars, $5 \mu \mathrm{m}$. Images in $\mathbf{g}-\mathbf{k}$ are representative of at least two independent experiments with similar results. Data are mean and single points; unpaired two-tailed Student $t$-tests. Source data and image quantifications are provided in Supplementary Table 3.

metabolic shift, we tested an involvement of the Hippo pathway. YAP/TAZ are inhibited by reduced actomyosin contractility ${ }^{3,4}$, but their knockdown did not cause lipid accumulation comparable to ROCK/MLCK inhibition (Supplementary Fig. 2a). Similarly, stable TAZ-4SA active mutant expression was not sufficient to prevent lipid accumulation upon ROCK/MLCK inhibition (Supplementary Fig. 2b). LATS1/2 (large tumour suppressor kinases 1 and 2) Hippo kinases can be activated by reduced actomyosin contractility ${ }^{10}$, but genetics indicate that LATS2 inhibits lipid metabolism ${ }^{11}$, which is incompatible with our results. This suggested the regulation of an alternative pathway, which we sought to identify by performing microarray analysis of MCF10ATk1 cells treated with Y27632+ ML7, followed by gene list enrichment analysis. As shown in Fig. 2a,b we found a striking enrichment of signatures related to cholesterol and fatty acid synthesis among the upregulated genes, including many known SREBP1 and SREBP2 (sterol regulatory element binding proteins) targets, pointing to activation of these transcription factors ${ }^{12,13}$. We thus validated activation of SREBP1/2 by a luciferase reporter for SREBP transcriptional activity, by qPCR, and in multiple cell lines (Fig. 2c,d and Supplementary Fig. 2d-f). Importantly, we also observed a coherent induction of SREBP target genes in cells cultured on soft hydrogels (Fig. 2e-g), while YAP/TAZ inhibition had no effects on SREBP activity (Supplementary Fig. 2g,h). Of note, among the validated SREBP targets LDLR (low density lipoprotein receptor) expression correlated with increased fatty acid uptake (Supplementary Fig. 2i) and ACSS2 (acyl-CoA synthetase short chain 
a

\begin{tabular}{l} 
Reactome 2016 \\
\hline Cholesterol biosynthesis \\
\hline Regulation of cholesterol biosynthesis by SREBP \\
\hline Activation of gene expression by SREBP \\
\hline Metabolism of lipids and lipoproteins \\
\hline Fatty acid, triacylglycerol metabolism \\
\hline Macroautophagy \\
\hline PI3K cascade \\
\hline Downstream of signalling of activated FGFR1 \\
Signalling by FGFR1 \\
\hline PPARA activates gene expression
\end{tabular}
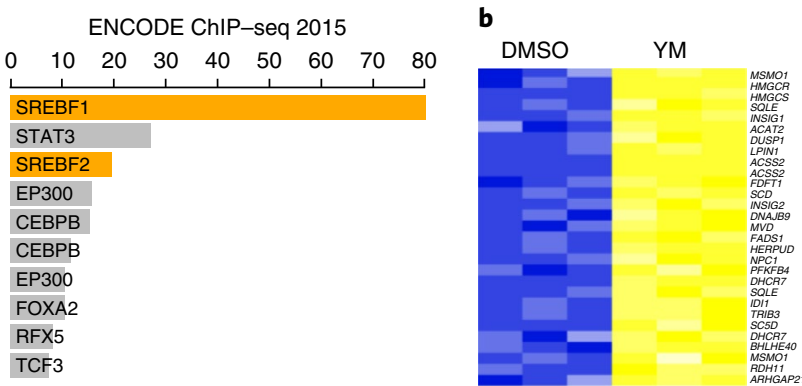

c

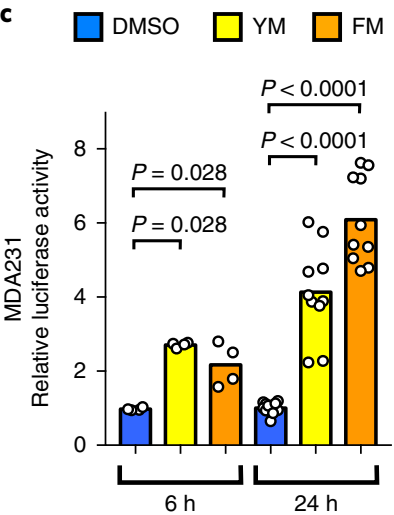

$\square$ DMSO $\square$ YM $\square$ FM
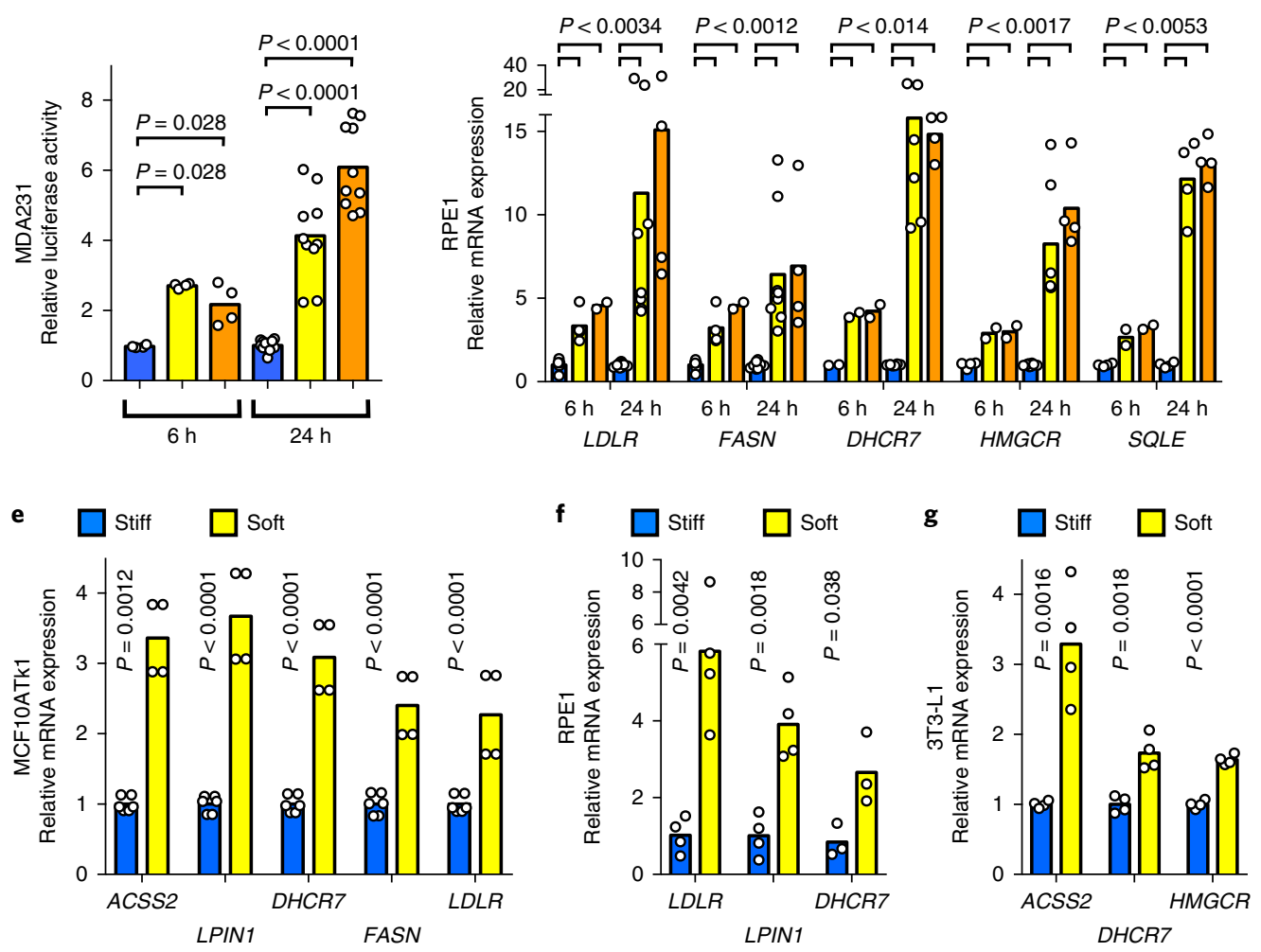

Fig. 2 | ECM mechanical cues regulate SREBP1/2 target genes. a, Gene list enrichment analysis on probes significantly upregulated (mean fold $>1.3$, $P<0.05)$ in microarrays of MCF10ATk1 cells treated with DMSO or Y27632+ML7 (YM) for $6 \mathrm{~h}$. The graphs display the 10 most significantly overrepresented gene sets for each of the indicated databases, analysed with Enrichr and ranked according to combined score ( $x$ axis). Gene sets related to cholesterol, lipids and SREBP are highlighted in orange. $\mathbf{b}$, Heatmap of SREBP target genes in microarrays of MCF10ATk1 cells treated as in $\mathbf{a}$. Each column is an independent biological sample ( $n=3$ for each condition). Each line corresponds to a single gene probe, indicated on the right. Blue and yellow extremes correspond to raw $Z$-scores of -2 and +2 , respectively. Microarray data are from a single experiment. c, $L D L R$-luciferase reporter assay for SREBP activity in MDA231 cells treated with YM or Fasudil+ML7 (FM). Mean expression in controls was set to 1 , and other samples are relative to this ( $n=4$ biologically independent samples pooled across two independent experiments for bars 1-3; $n=10$ biologically independent samples pooled across five independent experiments for bars 4-6; unpaired Mann-Whitney tests). d, qPCR for established SREBP targets in RPE1 cells treated for 6 or $24 \mathrm{~h}$ with DMSO, YM or FM ( $n=4$ biologically independent samples pooled across two independent experiments for each bar; multiple unpaired two-tailed Student's $t$-tests). e-g, qPCR for established SREBP targets in MCF10ATk1 (e), RPE1 (f) or 3T3L1 (g) cells plated on stiff ( $E \approx 15 \mathrm{kPa})$ or soft $(E \approx 0.5 \mathrm{kPa})$ ECM-coated hydrogels for $24 \mathrm{~h}$ ( $n=4$ biologically independent samples pooled across two independent experiments for each bar; multiple unpaired two-tailed Student's t-tests). In $\mathbf{d - g}$, data are relative to GAPDH levels; mean expression in controls (DMSO or stiff) were set to 1, and all other samples are relative to this. Data are mean and single points. All $n$ values are pooled between independent experiments. Source data are provided in Supplementary Table 3.

family member 2) with increased usage of acetate for lipid synthesis ${ }^{14}$ (Supplementary Fig. 2j).

ECM mechanical cues control lipid synthesis through direct regulation of SREBP1/2 activity. SREBP are produced as transmembrane proteins resident in the endoplasmic reticulum (ER) (see model in Supplementary Fig. 3a). In the presence of sterols and fatty acids, SCAP (SREBP cleavage-activating protein) and
Insig (insulin-induced gene) proteins bind SREBP and limit their transport to the Golgi apparatus. In the absence of lipids, conformational changes in SCAP and Insig enable the quantitative transport of SCAP and SREBP to the Golgi, where SREBP are processed by the S1P (site-1 protease) and S2P (site-2 protease) Golgi-resident enzymes. This in turn releases the cytoplasmic portion of SREBP that is free to accumulate into the nucleus to regulate gene transcription ${ }^{12,13}$. 

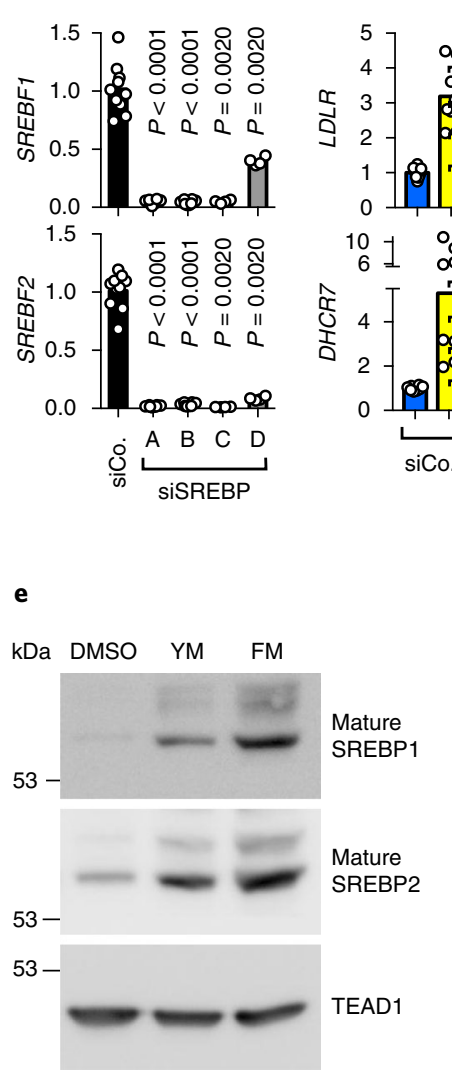

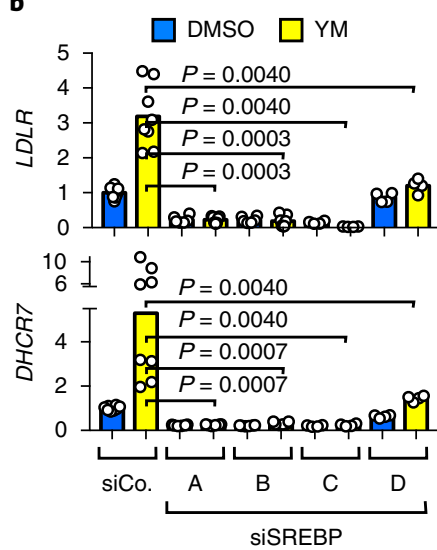

C

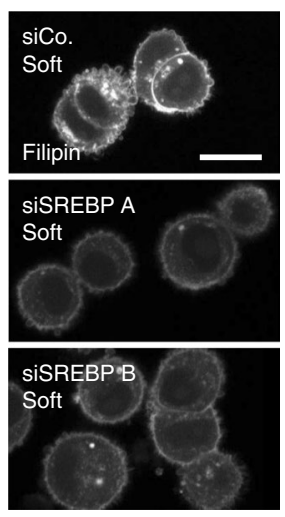

d
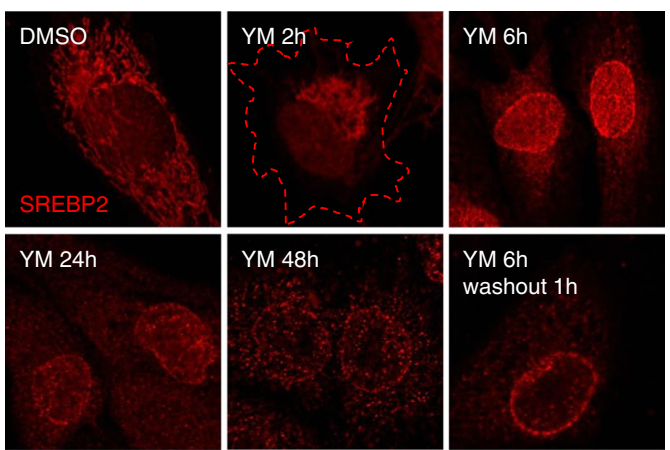

YM 48h
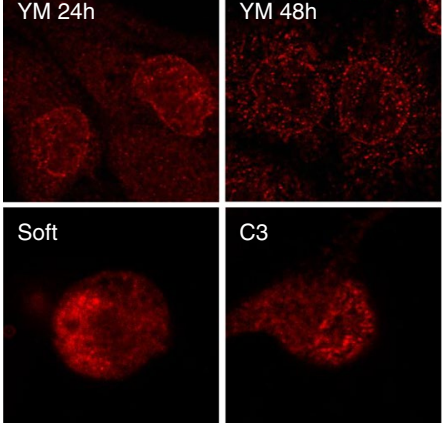

YM $6 \mathrm{~h}$ washout $1 \mathrm{~h}$
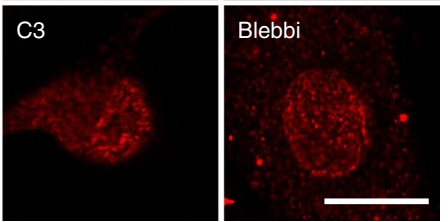

\section{f}
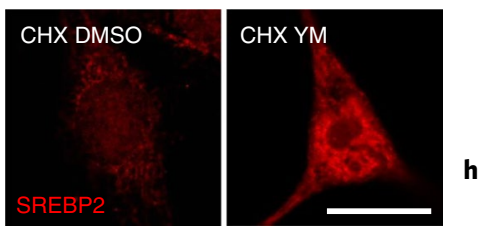

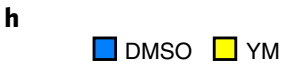

i Keloid scars versus normal skin \#1 \#2 \#3 \#4 \#5 \#6 \#7

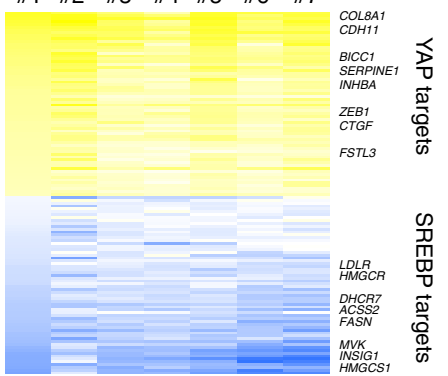

Fig. 3 | ECM mechanical cues regulate lipid synthesis by controlling SREBP1/2 activation. a, qPCR analysis in MCF10ATk1 cells $48 \mathrm{~h}$ after transfection with control siRNA (siCo.) or four independent mixes of siRNAs targeting SREBF1 and SREBF2 mRNAs (siSREBP A to D). b, qPCR analysis of SREBP1/2 targets in MCF10ATk1 transfected as in $\mathbf{a}$ and treated with DMSO or YM for $24 \mathrm{~h}$. In $\mathbf{a}$ and $\mathbf{b}$ mRNA expression data are relative to GAPDH levels; mean expression levels in controls were set to 1 , and all other samples are expressed relative to this $(n=10$ biologically independent samples pooled across five independent experiments for siCo.; $n=8$ biologically independent samples pooled across four independent experiments for $A$ and B; $n=4$ biologically independent samples pooled across two independent experiments for $C$ and $D$; unpaired Mann-Whitney tests). c, Cholesterol accumulation in MCF10ATk1 cells transfected with SREBP1/2 siRNA (siSREBP mixes A and B) and plated on soft hydrogels. Scale bar, $5 \mu \mathrm{m}$. $\mathbf{d}$, Immunofluorescence for endogenous SREBP2 in MCF10ATk1 cells with inhibited ROCK/MLCK (YM), RHO (C3 plasmid transfection), Myosinll (blebbistatin $10 \mu \mathrm{M}$ ) or plated on soft hydrogels for $6 \mathrm{~h}$. The cell contour in the YM $2 \mathrm{~h}$ panel (dashed line) helps visualize SREBP2 concentration at the Golgi apparatus. Scale bar, $10 \mu \mathrm{m}$. At least 50 cells per condition. e, Western blotting for the mature form of endogenous SREBP1 and SREBP2 in MCF10ATk1 cells treated for $4 \mathrm{~h}$ with YM or FM. TEAD1 is a loading control for nuclear extracts. f, Immunofluorescence for endogenous SREBP2 in MCF10ATk1 cells treated for $4 \mathrm{~h}$ with YM in the presence of $100 \mu \mathrm{g} \mathrm{ml}^{-1} \mathrm{cycloheximide}^{-1}$ $(\mathrm{CHX})$. Scale bar, $10 \mu \mathrm{m}$. At least 50 cells per condition. $\mathbf{g}$, Western blotting on total extracts of MCF10ATk1 cells untreated $(-)$, treated for $3 \mathrm{~h}$ with $2 \mu \mathrm{g} \mathrm{ml}^{-1}$ puromycin alone (puro) or with puromycin and $100 \mu \mathrm{g} \mathrm{ml}^{-1}$ cycloheximide (CHX). Incorporation of the puromycin amino acid analogue into nascent proteins is used as a control for efficient inhibition of protein synthesis. $\mathbf{h}, L D L R$-luciferase in MDA231 cells. YM and FM activate endogenous SREBP, but have no additive effects in cells depleted of SREBP1/2 (siSREBP) and expressing a siRNA-insensitive, cleaved mature SREBP2 cDNA (caSREBP2). Mean expression in controls was set to 1 , and other samples are relative to this $(n=4$ biologically independent samples pooled across two independent experiments for each bar; unpaired Mann-Whitney tests). $\mathbf{i}$, Heatmap of SREBP and YAP target levels in $n=7$ patient-matched soft normal skin versus stiff keloid tissue. Each column represents $-\log _{2}$ (keloid/skin) values for a single patient; each line is a single gene probe; genes ranked according to expression in patient \#1. Selected gene names are indicated on the right; only the 60 most up- or downregulated genes $(P<0.05)$ are included. Images in c-g are representative of at least two independent experiments with similar results. Data are mean and single points. All $n$ values are pooled between independent experiments. Unprocessed blots are provided in Supplementary Fig. 8. Source data and image quantification are provided in Supplementary Table 3.

Prompted by the observation that mechanical cues regulate SREBP activity, we sought to causally link SREBP activity with the effects of mechanical cues. As shown in Fig. 3a-c and Supplementary Fig. 3b,c, knockdown of SREBP1/2 inhibited expression of lipid enzymes and lipid accumulation in response to Y27632+ML7 and to a soft hydrogel. We then probed endogenous SREBP2 localization and observed an early concentration to the Golgi apparatus $(2 \mathrm{~h})$, closely followed by nuclear accumulation (4-6h), on treatment with ROCK/MLCK inhibitors (Fig. 3d and Supplementary
Fig. 3d-g). Nuclear accumulation of SREBP2 started to fade at $24 \mathrm{~h}$ and became almost undetectable at $48 \mathrm{~h}$ of treatment (Fig. 3d), probably due to accumulation of lipids eventually restraining SREBP2 activation. Moreover, nuclear accumulation was reversible, because washout of the inhibitors led to the rapid disappearance of nuclear SREBP2 (Fig. 3d). In keeping with a general effect of mechanical cues, nuclear SREBP2 was observed on transfection of the C3 RHO inhibitor, treatment with blebbistatin, and on soft hydrogels (Fig. 3d and Supplementary Fig. 3f,g). This was 
f
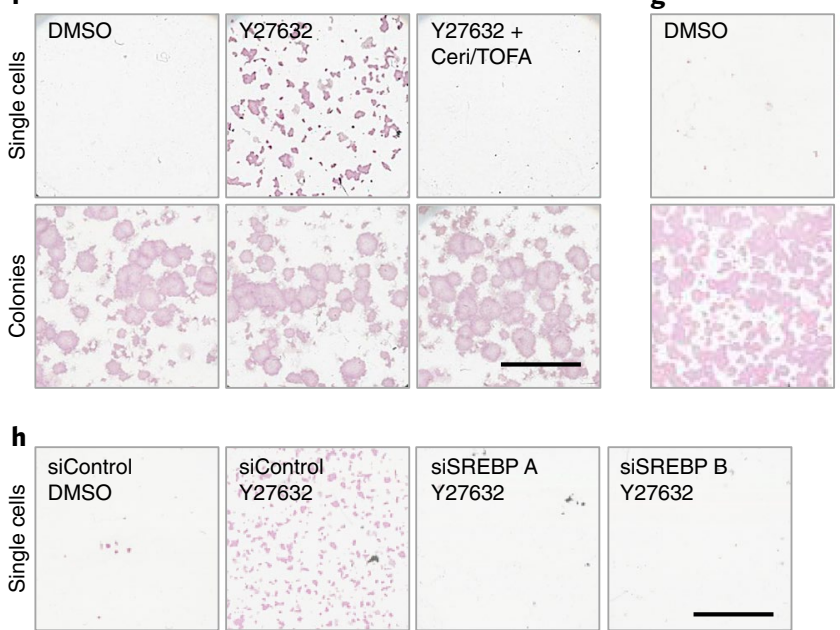
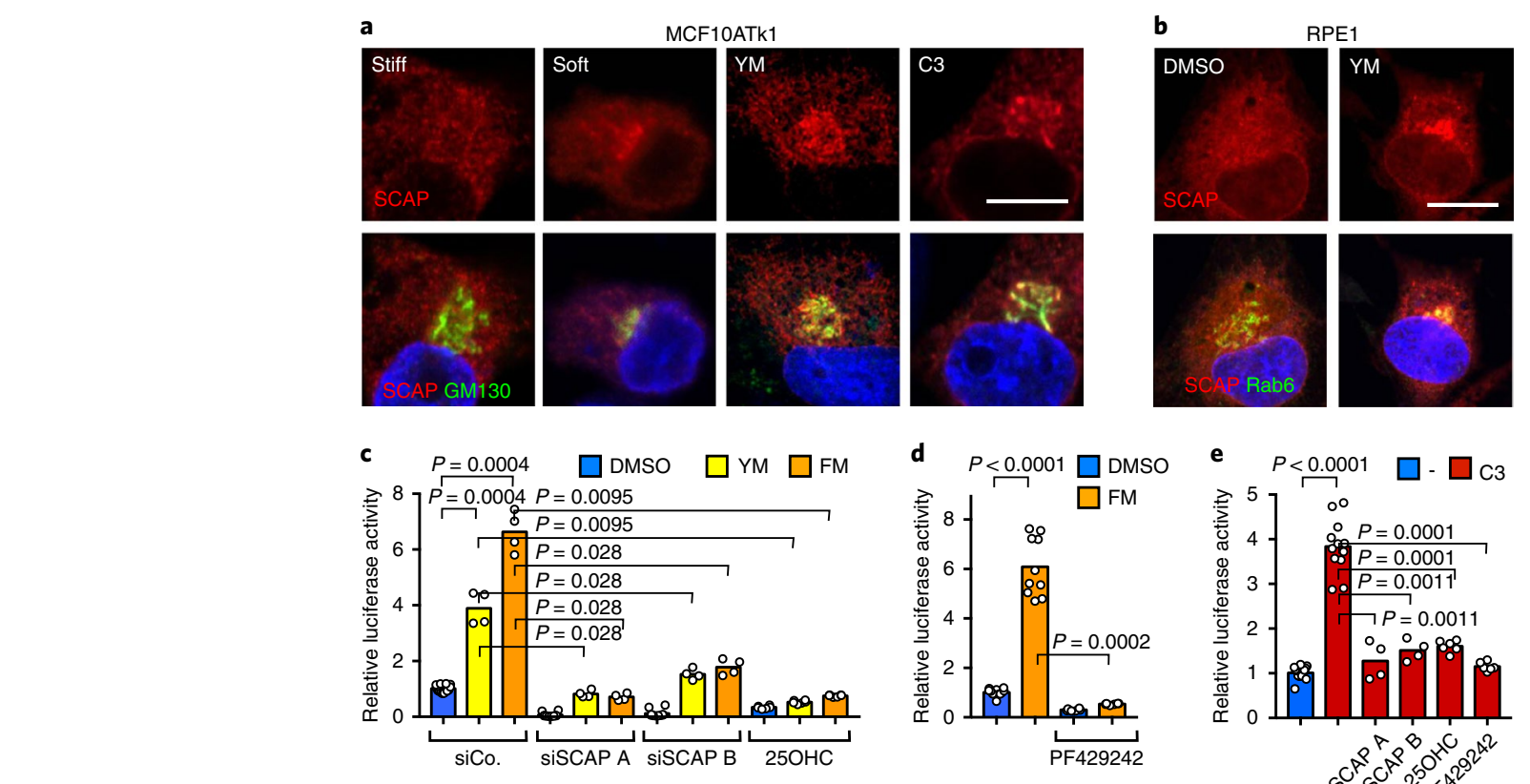

b RPE1
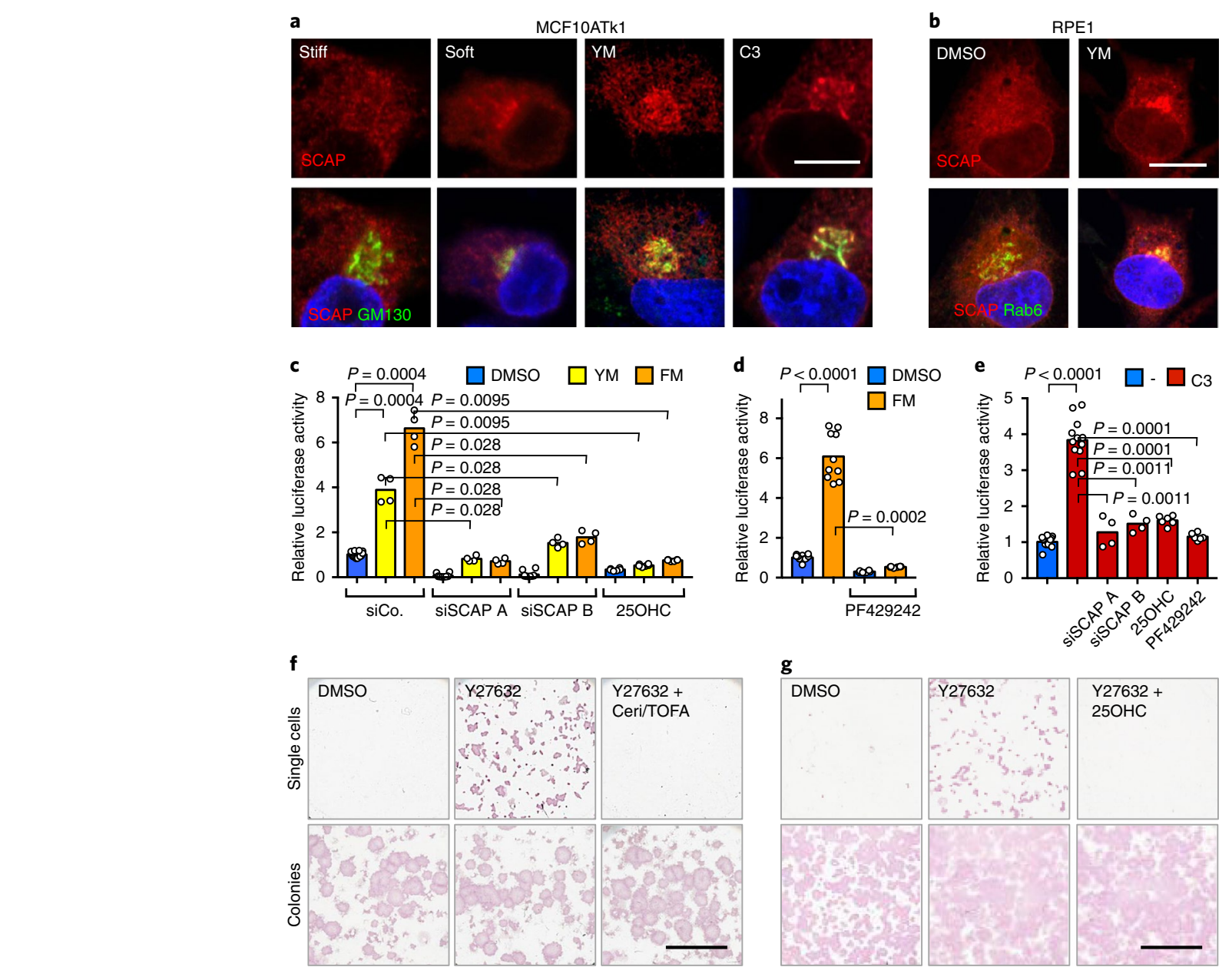

(1)
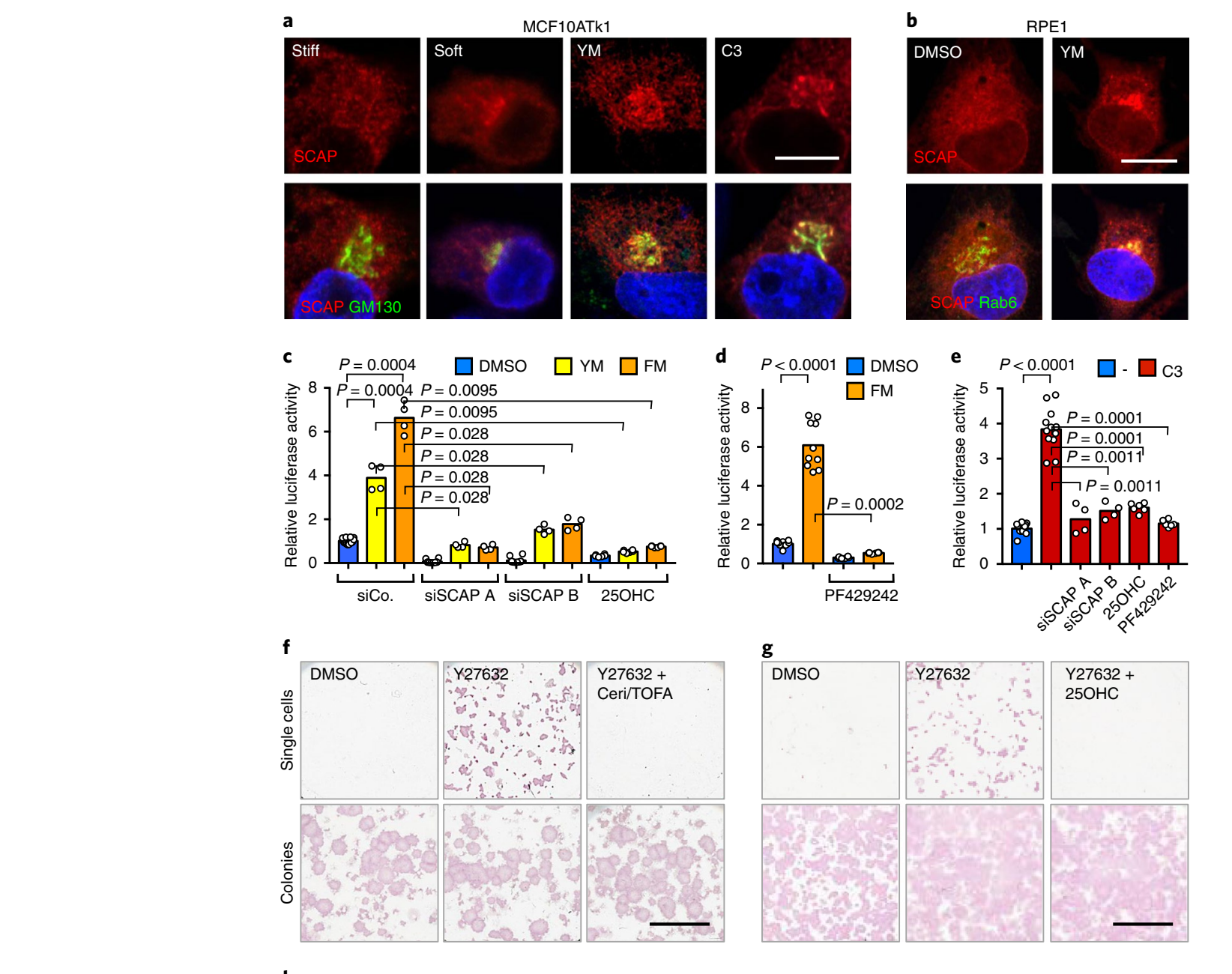

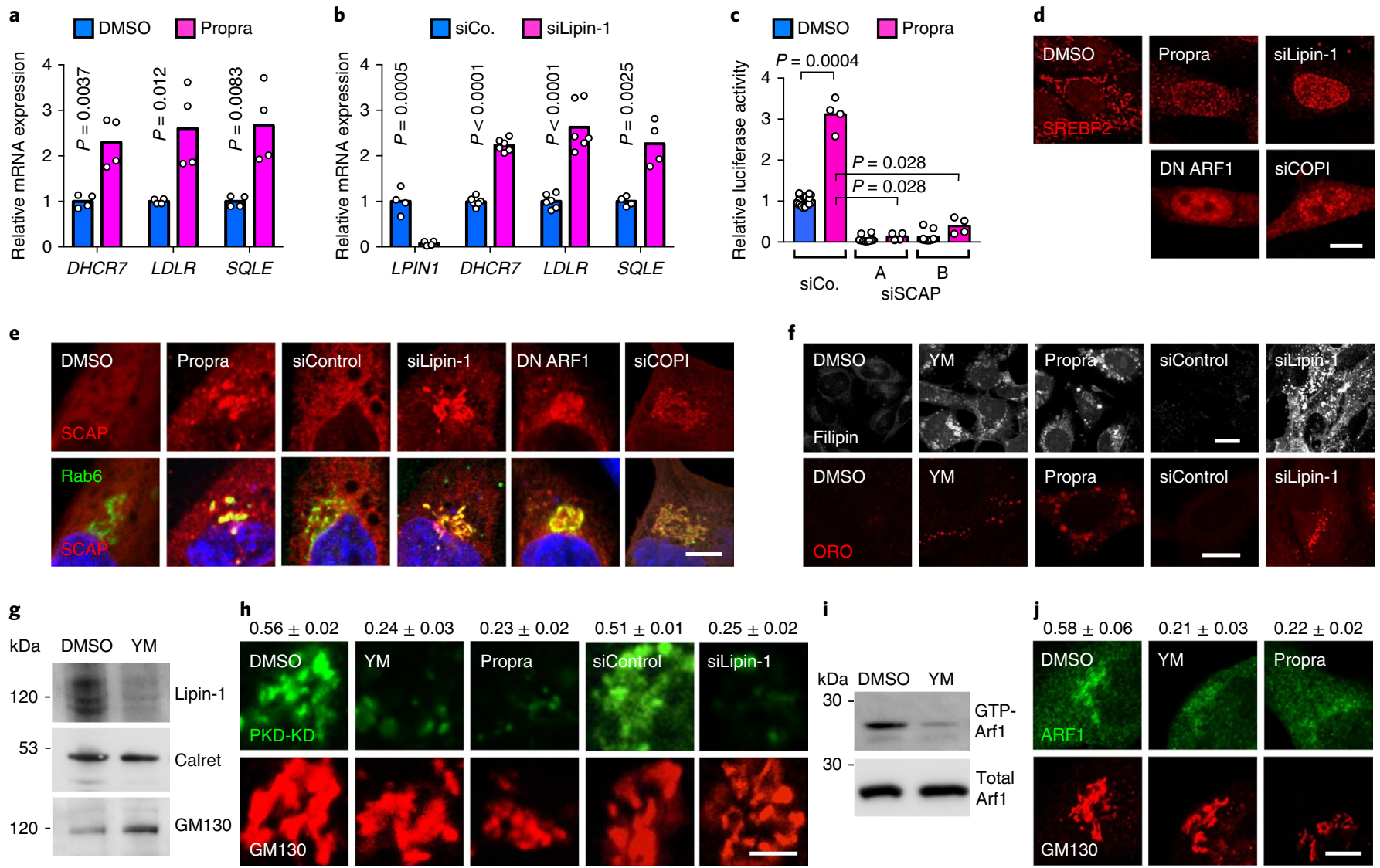

h
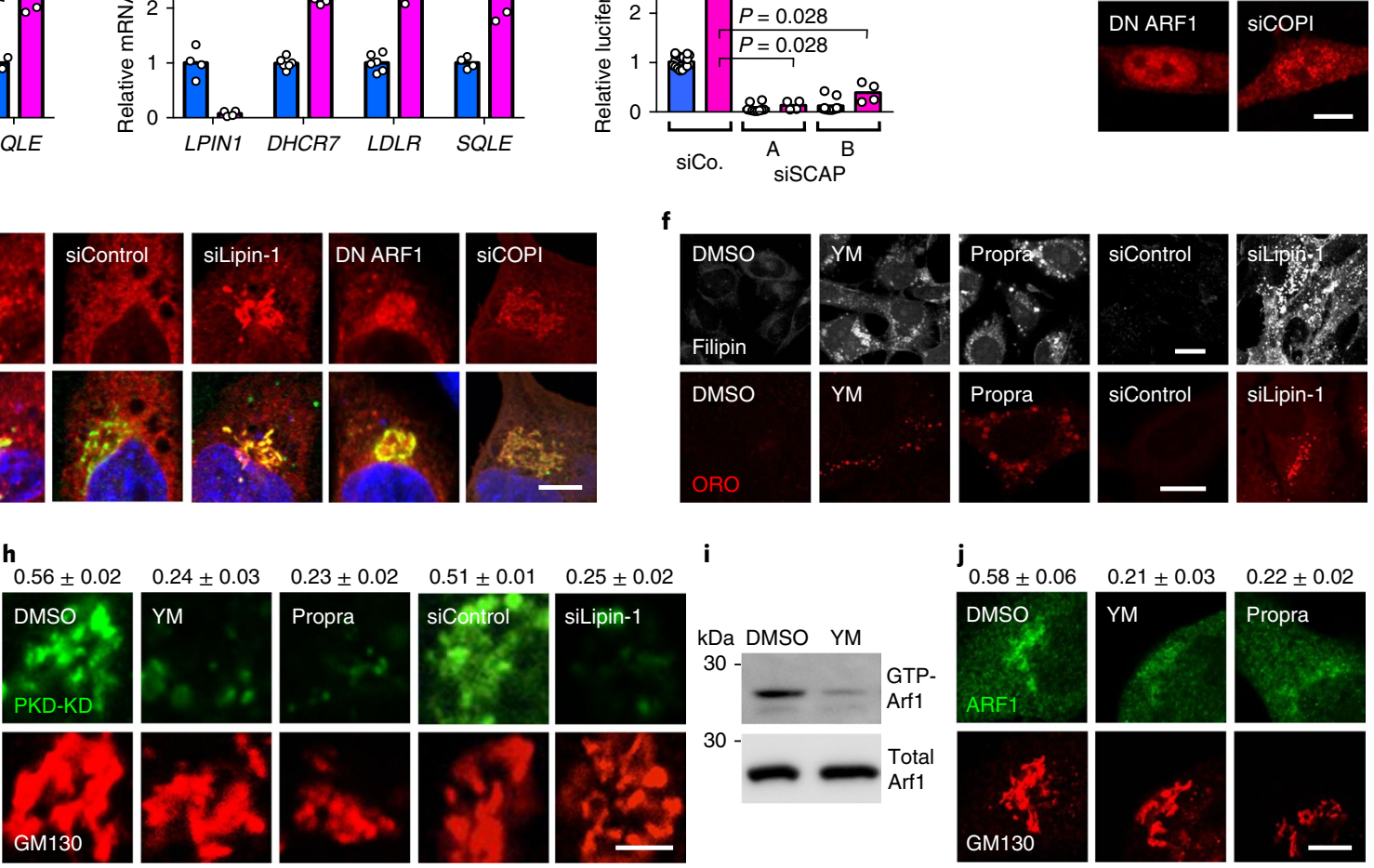

Fig. 5 | ECM mechanical cues regulate Lipin-1/ARF1 activity. a,b, qPCR for SREBP target genes in MCF10ATk1 cells treated with DMSO or with $100 \mu$ M propranolol (Propra) to inhibit Lipin-1 phosphatidate phosphatase activity (a), or transfected with control (siCo.) and Lipin-1 siRNA (siLipin-1) (b). Data are relative to GAPDH levels; mean expression in controls was set to 1 , and all other samples are relative to this ( $n=4$ biologically independent samples, except DHCR7 and LDLR in b for which $n=6$, pooled across two independent experiments for each bar; multiple unpaired two-tailed Student's $t$-tests). c, LDLR-luciferase in MDA231 cells transfected with the indicated siRNA and treated with $100 \mu \mathrm{M}$ propranolol. Mean expression in the control was set to 1, and all other samples are relative to this $(n=4$ biologically independent samples pooled across two independent experiments for each bar; unpaired MannWhitney tests). d, Immunofluorescence for endogenous SREBP2 in MCF10ATk1 cells treated with propranolol, transfected with Lipin-1 or COPI siRNA, or expressing dominant-negative ARF1-T31N-GFP (DN ARF1). At least 50 cells per condition. e, Co-localization of transfected MYC-SCAP with the GFP-Rab6 Golgi marker in RPE1 cells treated for $6 \mathrm{~h}$ with propranolol, in cells transfected with control (siCo.), Lipin-1 or COPI siRNAs, or expressing dominant-negative ARF1-T31N (DN ARF1). At least 50 cells per condition. f, Lipid staining in MCF10ATk1 cells treated for $24 \mathrm{~h}$ with YM, with $100 \mu \mathrm{M}$ propranolol, or transfected with Lipin-1 siRNA. Quantifications and $n$ are provided in Supplementary Table 3. $\mathbf{g}$, Western blotting for Lipin-1 levels in microsomal fractions from MCF10ATk1 cells treated for $3 \mathrm{~h}$ with DMSO or Y27632+ML7 (YM). Calreticulin (ER marker, Calret) and GM130 (Golgi marker) are loading controls. h, Co-localization of transfected GFP-PKD-KD with the GM130 Golgi marker in HEK293 cells treated for 30 min with YM, with $100 \mu$ M propranolol, or transfected with control (siCo.) and Lipin-1 siRNAs. Mean Pearson's correlation coefficient and s.d. for co-localization are indicated above each panel ( $n \geq 10$ cells were measured for each condition). i, GST-GGA3-PBD pulldown for GTP-bound active ARF1 (GTP-Arf1) and western blotting for ARF1 in the total extracts (Total Arf1). Cells were treated 3h with DMSO or YM. j, Co-localization of endogenous ARF1 with a Golgi marker (GM130) in MCF10ATk1 cells treated $6 \mathrm{~h}$ with YM or propranolol. Mean Pearson's correlation coefficient and s.d. for co-localization are indicated above each panel ( $n \geq 10$ cells were measured for each condition). Images in $\mathbf{d}$-j are representative of at least two independent experiments with similar results. Scale bars, $10 \mu \mathrm{m}$, except for $\mathbf{f}(5 \mu \mathrm{m})$. Data are mean and single points. All $n$ values are pooled across independent experiments. Unprocessed blots are provided in Supplementary Fig. 8.

Inhibition of SREBP activity by pathological tissue stiffness. To find evidence that this regulation also occurs in vivo, we queried gene expression data obtained by comparing patient-matched normal skin with keloid scars ${ }^{15}$, a fibroproliferative disorder characterized by increased tissue stiffness and whose expansion is linked to mechanical stress ${ }^{16,17}$. Strikingly, several SREBP target genes were consistently and uniformly downregulated in stiffened keloids across all patients, supporting our model (Fig. 3i). Of note, this finding nicely parallels the so far unexplained decrease in lipids and cholesterol-esters observed in keloids ${ }^{18}$. As a control, we also found upregulation of several YAP/TAZ target genes (Fig. 3i), in line with stiffness-induced YAP/TAZ activity during fibrosis ${ }^{19,20}$.
Thus, cytoskeletal tension is a relevant input to regulate SREBP in vitro and in at least one human pathological tissue.

ECM mechanical cues regulate SCAP localization and function. We next probed the subcellular localization of core SREBP regulators. While Insig1 and S1P remained correctly confined to the ER and the Golgi apparatus, respectively (Supplementary Fig. 4a,b), we observed translocation of SCAP to the Golgi apparatus by plating cells on soft hydrogels and after inhibition of RHO or ROCK/MLCK (Fig. 4a,b and Supplementary Fig. 4c). This was instrumental for the regulation of SREBP by mechanical cues, because both SCAP knockdown and treatment of cells with 25-hydroxycholesterol, 
a

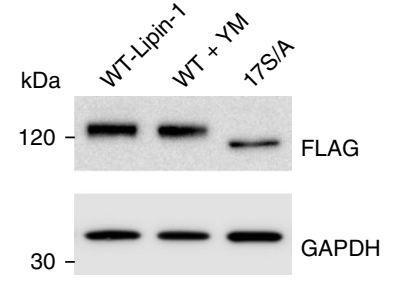

d
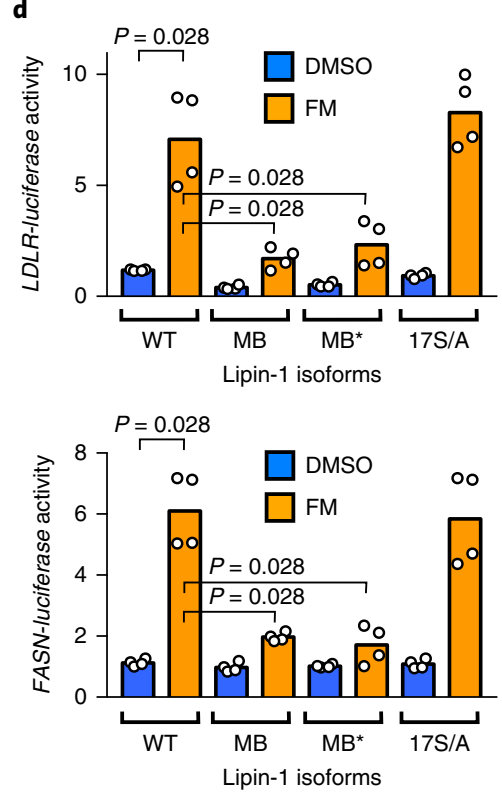

b

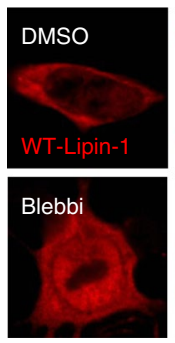

e
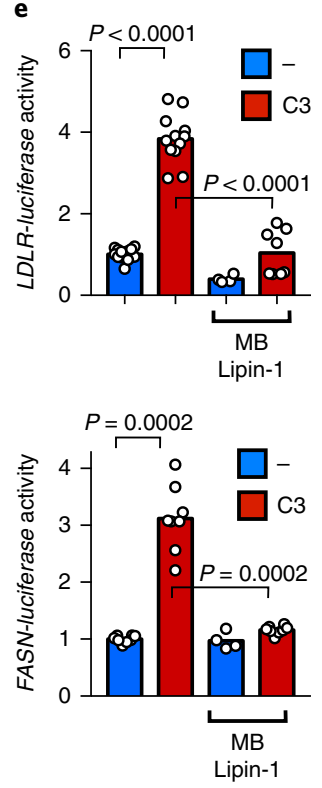

c
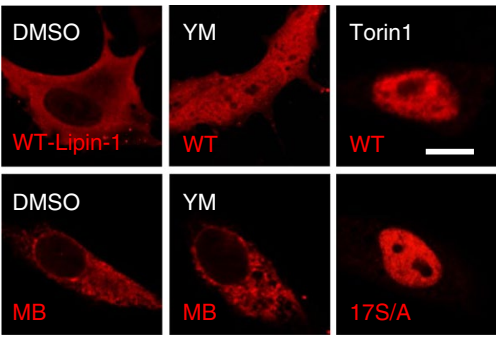

f


Fig. 6 | Actomyosin contractility regulates SREBP through Lipin-1. a, Western blotting for transfected FLAG-tagged Lipin-1 in HEK293 cells treated with DMSO or YM for $6 \mathrm{~h}$. The 17S/A mutant is not phosphorylated and migrates faster than WT Lipin-1. GAPDH serves as loading control.

b, Immunofluorescence for transfected FLAG-Lipin-1 in MCF10ATk1 cells treated $6 \mathrm{~h}$ with YM, blebbistatin, or plated on a soft hydrogel. Scale bar, $10 \mu$ m. At least 50 cells per condition. c, Immunofluorescence for transfected wild-type (WT) or 17S/A FLAG-tagged Lipin-1 isoforms in RPE1 cells, treated with DMSO, YM or Torin1 (500 nM) for $6 \mathrm{~h}$. Fusion with the membrane-localization domain of Syntaxin5 tethers MB-Lipin-1 to cytoplasmic membranes. Scale bar, $10 \mu \mathrm{m}$. At least 50 cells per condition. Similar results were obtained with MB*-Lipin-1 (used in d) and in other cell lines. $\mathbf{d}$, LDLR-luciferase (top) and FASN-luciferase (bottom) assays in MDA231 cells treated with the ROCK/MLCK inhibitor FM and transfected with WT, membrane-tethered $\left(\mathrm{MB}\right.$ and $\mathrm{MB}^{\star}$ ) or 17S/A Lipin-1 isoforms ( $n=4$ biologically independent samples pooled across two independent experiments for each bar; unpaired Mann-Whitney tests). e, LDLR-luciferase (top) and FASN-luciferase (bottom) assays in MDA231 cells transfected with the C3 RHO inhibitor without or with membrane-tethered (MB) Lipin-1 ( $n \geq 6$ biologically independent samples pooled across three independent experiments for bars 1,2 and $4 ; n=4$ biologically independent samples pooled across two independent experiments for bar 3; unpaired Mann-Whitney tests). In d and e, mean expression in the control was set to 1, and all other samples are relative to this. $\mathbf{f}$, MDA231 cells were transfected with WT-Lipin-1 or MB-Lipin-1 together with mCherry (RFP), treated with YM, and stained for cholesterol. At least 30 cells per condition. Bottom, Cells were scored positive (+ve) for Filipin based on the presence/absence of cytoplasmic cholesterol accumulation. Images in $\mathbf{a}-\mathbf{c}$ and $\mathbf{f}$ are representative of at least two independent experiments with similar results. Data are mean and single points. All $n$ values are pooled across independent experiments. Source data are provided in Supplementary Table 3. Unprocessed blots are provided in Supplementary Fig. 8.

a dominant inhibitor of SCAP transport ${ }^{21}$, decreased SREBP activation (Fig. 4c,e and Supplementary Fig. 4d). In line, treating cells with the S1P inhibitor PF429242 also prevented activation of SREBP (Fig. 4d,e). This indicates that actomyosin contractility prevents accumulation of SCAP/SREBP to the Golgi apparatus and the exposure of SREBP to Golgi proteases.

Lipid synthesis contributes to the beneficial effects of ROCK inhibitors in hPSC. To expand the functional implications of these findings we used human pluripotent stem cells (hPSCs). These cells require treatment with the Y27632 ROCK inhibitor to survive single-cell dissociation, whereas they thrive without inhibitor once they have attached to the substrate ${ }^{22,23}$. We thus tested the idea that
Y27632 might promote survival of hPSCs by enhancing lipid synthesis. We first confirmed that Y27632 induces lipid accumulation also in these cells (Supplementary Fig. 4e). We then challenged Y27632induced survival of hPSCs seeded as single cells by using very low doses of lipid synthesis inhibitors (cerivastatin and TOFA), and found that hPSCs did not survive (Fig. 4f, single cells); the same dose of inhibitors was instead inconsequential for cell survival of already established hPSC colonies, when Y27632 is not required (Fig. 4f, colonies). In line, both treatment of cells with 25-hydroxycholesterol and transfection of SREBP1/2 siRNAs impaired Y27632-induced single-cell survival (Fig. 4g,h). These results suggest that isolated hPSC are highly dependent on lipid synthesis, and that ROCK inhibition promotes their survival, at least in part, by sustaining SREBP activity. 

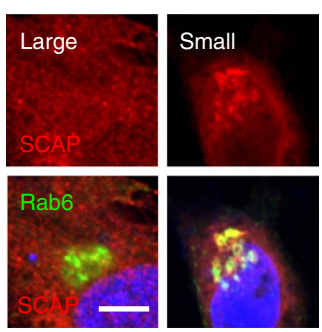

b

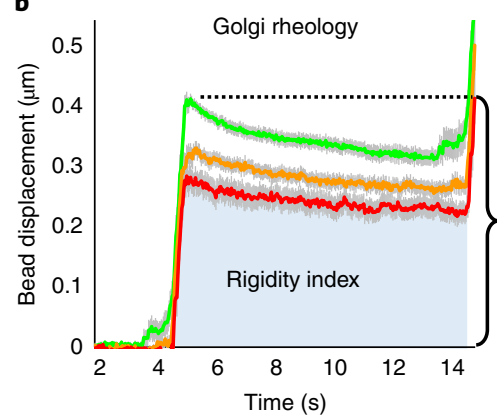

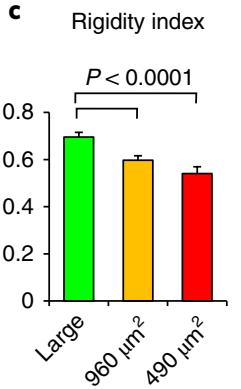

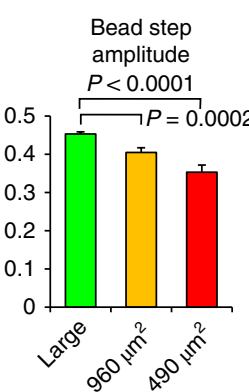

$$
\text { amplitude }
$$

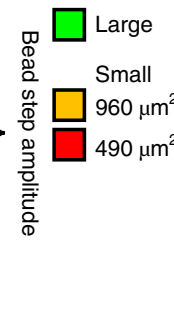

Ejection

frequency
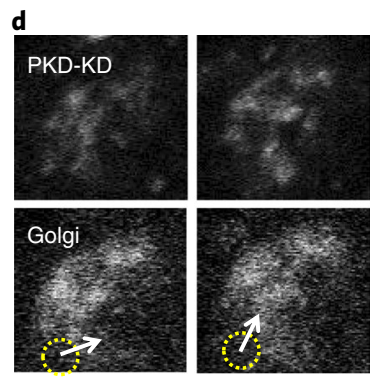

$t=0$

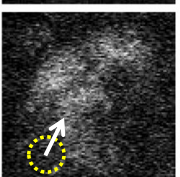

$t=10 \mathrm{~min}$

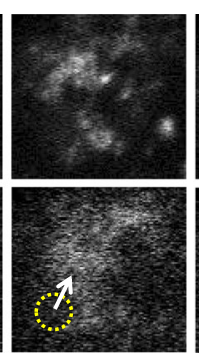

$t=20 \mathrm{~min}$

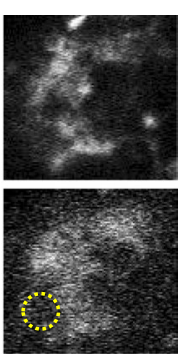

$t=30 \mathrm{~min}$

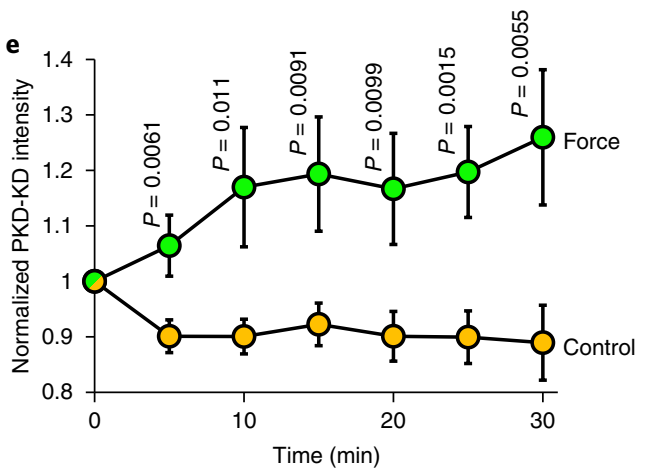

Fig. 7 | The Golgi apparatus responds to extracellular physical cues and intracellular force application. a, Co-localization of transfected MYC-SCAP with the GFP-Rab6 Golgi marker in RPE1 cells freely spreading on fibronectin-coated glass (Large) or plated on micropatterned fibronectin islands restraining cell area and inducing low F-actin tension (Small, $960 \mu \mathrm{m}^{2}$ or $490 \mu \mathrm{m}^{2}$ ). Scale bar, $10 \mu \mathrm{m}$. At least 30 cells per condition. $\mathbf{b}$, Golgi rheology was measured in RPE1 cells plated as in a. GFP-Rab6-positive Golgi membranes were pushed towards a cytoplasmic bead immobilized by an optical trap in a series of five $0.5 \mu \mathrm{m}$ steps in $1 \mathrm{~min}$ (Supplementary Fig. 7a and Methods). The graph shows the averaged displacements of the bead during the first step (see Supplementary Fig. $7 \mathrm{~b}$ for the complete graph). Green line: large unconfined cells, conditions of high tension ( $n=39$ cells). The Golgi microenvironment displays a visco-elastic behaviour as the bead is first maximally displaced (bead step amplitude), and then slowly relaxes back due to attraction from the optical trap. Orange and red lines: micropatterned small ECM $(n=28,21$ cells). Gray shading: s.e.m. error. A smaller displacement and a faster relaxation of the bead indicate a lower rigidity of the Golgi apparatus. c, The rigidity index measures the friction opposed by the Golgi microenvironment on the bead, with lower values indicating a softer microenvironment. The bead step amplitude corresponds to the average displacement of the bead after each step, with lower values indicating a softer microenvironment. The ejection frequency measures the relative frequency of experiments in which the bead falls off the optical trap, which is increased with the resisting friction forces applied on the bead by the Golgi. d, Time-lapse confocal images of a representative RPE1 cell transfected with the GFP-PKD-KD diacylglycerol sensor and with Golgi-localized mCherry. A 2- $\mu$ m-diameter cytoplasmic bead (yellow dotted circle) was immobilized by an optical trap in the proximity of the Golgi apparatus $(t=0)$ and then pushed every 5 min towards the Golgi (white arrow, direction of compressive constraint). e, Normalized intensity of the GFP-PKD-KD signal before and after application of force ( $n=19$ cells). In the control the bead was moved away from the Golgi towards the cytoplasm ( $n=18$ cells). Images in $\mathbf{a}$ and $\mathbf{d}$ are representative of at least two independent experiments with similar results. Data are mean and s.e.m.; two-tailed unpaired Student's $t$-tests. All $n$ values are pooled across independent experiments. Source data are provided in Supplementary Table 3.

Uncoupling actomyosin contractility from intracellular cholesterol trafficking. One possibility to explain our findings is that reduced actomyosin contractility leads to reduced levels of sterols at the ER, because of decreased transport of extracellular cholesterol from the endosome/lysosome to the ER by the NPC1 transporter ${ }^{12,13}$. We excluded this scenario based on the following observations: (1) cholesterol accumulation in response to Y27632+ML7 was decreased in cells with inhibited synthesis or with SREBP1/2 knockdown (see Figs 1g, 3c and Supplementary Fig. 3c), indicating it is a secondary effect; (2) cholesterol accumulation occurred after SREBP activation, and with a delayed kinetics compared to inhibition of the NPC1 transporter with U18666A (Supplementary Fig. 5a); (3) while U18666A induced accumulation of cholesterol in LAMP2-encircled lysosomal structures, as expected, only a few Y27632+ML7-induced cholesterol dots were positive for LAMP2 (Supplementary Fig. 5b); (4) treatment with U18666A or serum (and, thus, LDL-cholesterol) deprivation caused concentration of the ER cholesterol-sensing protein OSBP to the Golgi apparatus at early time points ${ }^{24,25}$, unlike treatment with Y27632+ML7 (Supplementary Fig. 5c).
Linking actomyosin contractility to the Lipin-1/ARF1 SREBPregulatory axis. Looking for alternative mechanisms, we reasoned that among the known SREBP-regulatory inputs, two act independently of sterol levels: ARF1 and Lipin-1. The ARF1 (ADP ribosylation factor 1) small $G$ protein regulates Golgi dynamics ${ }^{26,27}$ and inhibits SREBP ${ }^{28-30}$. The precise mechanism remained incompletely understood, because Walker and colleagues ${ }^{28}$ have shown that ARF1 prevents S1P from shuttling to the ER, while Nakayama and colleagues $^{30}$ have shown that the ARF1 effector COPI (coat protein complex I) prevents accumulation of SCAP/SREBP to the Golgi. Lipin-1 is a phosphatase that converts phosphatidates into diacylglycerols at cytoplasmic membranes ${ }^{31}$, and also an inhibitor of SREBP activity $^{29,32}$. Interestingly, ARF1 recruitment to the Golgi apparatus and formation of COPI-coated vesicles requires diacylglycerols ${ }^{33-36}$, such that inhibition of Lipin-1 activity can cause ARF1 dissociation from Golgi membranes and ARF1 inhibition ${ }^{29,35}$. This suggested that reduced actomyosin contractility might induce SREBP activity by inhibiting Lipin-1/ARF1 (see model in Supplementary Fig. 5d).

We first verified that inhibition of Lipin-1, ARF1 or COPI induce SREBP activation, SCAP accumulation at the Golgi apparatus and 
lipid accumulation in our systems (Fig. 5a-f and Supplementary Fig. 5e). We then indirectly gauged Lipin-1 activity by monitoring its association with microsomes ${ }^{37,38}$ or by monitoring Golgi membrane DG content with the DG-binding domain of PKD1 (GFPPKD-KD $)^{35,39,40}$, and found that inhibition of ROCK/MLCK rapidly caused Lipin-1 dissociation from microsomes (Fig. $5 \mathrm{~g}$ ) and decreased GFP-PKD-KD co-localization with the Golgi apparatus (Fig. 5h). ROCK/MLCK inhibition also decreased ARF1 activity (Fig. 5i) and ARF1 recruitment to the Golgi apparatus (Fig. 5j). Moreover, both Lipin-1 and ROCK/MLCK inhibition induce similar remodelling of Golgi morphology at late time points (Supplementary Fig. 5f,g), a phenotype previously observed in cells depleted of GBF1/ARF1 ${ }^{28}$. We did not observe a general redistribution of a fluorescent KDEL reporter (Supplementary Fig. 5h), indicating overall intact transports between ER and Golgi. Collectively, our data indicate that mechanical cues control Lipin-1 activity, causing altered ARF1-dependent trafficking of SCAP/SREBP between the Golgi and the ER.

Actomyosin contractility controls SREBP through Lipin-1, but independently from AMPK/mTOR. We then explored how Lipin-1 is regulated. Lipin-1 activity and association to cytoplasmic membranes can be inhibited by phosphorylation ${ }^{32,37,38}$. We checked Lipin-1 overall phosphorylation levels, but we did not observe major changes of the Lipin-1 migratory pattern (Fig. 6a). We also monitored Lipin-1 subcellular localization by immunofluorescence, and found that reduced contractility partially shifted FLAG-Lipin-1 towards the nucleus (Fig. 6b,c). Nuclear Lipin-1 has been associated with inactivation of $\mathrm{SREBP}^{32}$; because we observed a partial nuclear accumulation of Lipin-1 in conditions of active SREBP, we then wondered what pool of Lipin-1 was relevant in our cells. For this we compared wild-type Lipin-1 (WT) with a nuclear phospho-mutant (17S/A) and with Lipin-1 isoforms that we designed to constitutively associate with ER/Golgi membranes (MB-Lipin-1 and $\mathrm{MB}^{\star}$-Lipin-1; Fig. $6 \mathrm{c}$ ), and used these to challenge the effects of reduced contractility. While the WT and 17S/A mutant were inactive, expression of membrane-associated Lipin-1 counteracted SREBP activity (Fig. 6d,e) and lipid accumulation (Fig. 6f). Also, the SUMOylation $^{41}$ and acetylation ${ }^{42}$ Lipin- 1 mutants were inactive in the same assay (Supplementary Fig. 6a). Collectively, these results indicate that reduced contractility inhibits the affinity of Lipin-1 for ER/Golgi membranes, leading to nuclear localization of Lipin-1 as a secondary effect. This occurs independently from mTOR, a known Lipin-1 regulatory input (Supplementary Fig. 6b,c), and also from AMPK, a main metabolic regulator (Supplementary Fig. 6d,e).

The Golgi apparatus responds to extracellular and intracellular forces. We finally sought to obtain some insight into how extracellular mechanical cues influence signalling at the Golgi apparatus. Transmission of forces from the ECM entails activation of signalling molecules at focal adhesions ${ }^{1}$ but also occurs in a direct fashion, for example by stretching the plasma membrane, or owing to anchoring of stress fibres to the nuclear lamina ${ }^{43,44}$. Whether the Golgi apparatus might respond in a similar fashion has not been explored, but Golgi cisternae are embedded in a complex cytoskeletal network ${ }^{45,46}$, and the Golgi microenvironment is endowed with an intrinsic mechanical rigidity that depends on ROCK/MLCK ${ }^{47}$. To measure the response of Golgi rigidity to external physical cues we plated cells on small fibronectin-coated micropatterns, to which cells respond by decreasing actomyosin contractility ${ }^{48}$, and by accumulating SCAP to the Golgi apparatus (Fig. 7a). We then measured Golgi rheology by pushing on the Golgi apparatus a cytoplasmic bead immobilized in a laser optical trap (see Methods and Supplementary Fig. 7a). Analysis of the averaged relaxation curves (Fig. $7 \mathrm{~b}$ and Supplementary Fig. 7b) indicated a lower rigidity of the Golgi apparatus in micropatterned cells, apparent from a reduction of the bead step amplitude, the rigidity index and the frequency of bead ejection (Fig. 7c). As a control, moving the bead away from the Golgi showed that the overall cytoplasmic stiffness is not altered (Supplementary Fig. 7c). This experiment indicates that Golgi stiffness is coupled, directly or indirectly, to the mechanical properties of the ECM. Strikingly, with this set-up we also observed that direct application of force to the Golgi apparatus was sufficient to induce recruitment of the GFP-PKD-KD reporter (Fig. 7d,e), in line with higher Lipin-1 activity in cells developing higher contractile forces (see 'Linking actomyosin contractility to the Lipin-1/ARF1 SREBPregulatory axis' and Fig. 6).

\section{Discussion}

Seminal work demonstrating that cells sense ECM mechanical cues set the array of bona fide mechanoregulated phenotypes in vitro. Yet, what other general cell phenotypes are regulated by mechanical cues remains largely unexplored. Here, we have focused on cell metabolism and identified neutral lipid and cholesterol synthesis as a general response to reduced actomyosin contractility and to a soft ECM microenvironment. We propose that decreased extracellular forces reverberate on Golgi rheology and inactivate Lipin-1, causing alteration of diacylglycerol at the Golgi apparatus, reduced recruitment of ARF1 and ultimately leading to activation of SREBP1/2 transcription factors. As such, our data provide a missing upstream input for the Lipin-1 and ARF1 SREBP-regulatory axis, whose players were previously identified in Caenorhabditis elegans ${ }^{29}$, and validate the idea that continuous shuttling of SCAP/SREBP from the Golgi to the ER is a significant mechanism limiting their activa$\operatorname{tion}^{30}$. These findings led us to revisit the requirement of isolated hPSCs for ROCK inhibition, which we found linked to the ability to sustain SREBP activity and lipid synthesis. We speculate that this might underlie the beneficial effect of ROCK inhibition observed for isolation of other primary stem-cell populations $s^{49-51}$. These findings also indicate that physiological or pathological conditions leading to altered tissue stiffness may impact SREBP activity and lipid metabolism; strikingly, we could obtain a proof of principle for this in human keloid scars, providing a plausible explanation for reduced triglycerides and cholesteryl-esters ${ }^{18}$. Thus, we here identify an unexpected and widespread mechanoresponsive phenotype as well as its main underlying mechanism. Our findings raise interesting questions on how Lipin-1 is regulated in this context; we speculate that Lipin-1 macromolecular complexes observed on supported lipid bilayers in vitro ${ }^{52}$ are endowed with curvature-sensing ability, which might link Lipin-1 activity to the rheology of Golgi membranes and the cytoskeleton. In future, it will be interesting to test whether ECM mechanical cues affect other functions of the Golgi, of Lipin-1 signalling and of ARF1, and how these impact the responses of normal and diseased tissues to mechanical cues.

\section{Online content}

Any methods, additional references, Nature Research reporting summaries, source data, statements of data availability and associated accession codes are available at https://doi.org/10.1038/ s41556-018-0270-5.

Received: 19 April 2018; Accepted: 20 December 2018;

Published online: 04 February 2019

\section{References}

1. Iskratsch, T., Wolfenson, H. \& Sheetz, M. P. Appreciating force and shape-the rise of mechanotransduction in cell biology. Nat. Rev. Mol. Cell Biol. 15, 825-833 (2014).

2. LeGoff, L. \& Lecuit, T. Mechanical forces and growth in animal tissues. Cold Spring Harb. Perspect. Biol. 8, a019232 (2015).

3. Dupont, S. et al. Role of YAP/TAZ in mechanotransduction. Nature 474, 179-183 (2011).

4. Dupont, S. Role of YAP/TAZ in cell-matrix adhesion-mediated signalling and mechanotransduction. Exp. Cell Res. 343, 42-53 (2016). 
5. Miralles, F., Posern, G., Zaromytidou, A.-I. \& Treisman, R. Actin dynamics control SRF activity by regulation of its coactivator MAL. Cell 113, 329-342 (2003).

6. Janmey, P. A., Wells, R. G., Assoian, R. K. \& McCulloch, C. A. From tissue mechanics to transcription factors. Differentiation 86, 112-120 (2013).

7. Coloff, J. L. et al. Differential glutamate metabolism in proliferating and quiescent mammary epithelial cells. Cell Metab. 23, 867-880 (2016).

8. Bays, J. L., Campbell, H. K., Heidema, C., Sebbagh, M. \& DeMali, K. A. Linking E-cadherin mechanotransduction to cell metabolism through force-mediated activation of AMPK. Nat. Cell Biol. 19, 724-731 (2017).

9. Romani, P. et al. Metabolomics and lipidomics analyses of MCF10ATk1 cells treated with ROCK/MLCK inhibitors. Figshare https://doi.org/10.6084/m9. figshare.7338764 (2018).

10. Zhao, B. et al. Cell detachment activates the Hippo pathway via cytoskeleton reorganization to induce anoikis. Genes Dev. 26, 54-68 (2012).

11. Aylon, Y. et al. The LATS2 tumor suppressor inhibits SREBP and suppresses hepatic cholesterol accumulation. Genes Dev 30, 786-797(2016).

12. Shimano, H. \& Sato, R. SREBP-regulated lipid metabolism: convergent physiology-divergent pathophysiology. Nat. Rev. Endocrinol. 13, 710-730 (2017).

13. Brown, M. S., Radhakrishnan, A. \& Goldstein, J. L. Retrospective on cholesterol homeostasis: the central role of Scap. Annu. Rev. Biochem. 87, 783-807 (2018).

14. Comerford, S. A. et al. Acetate dependence of tumors. Cell 159, 1591-1602 (2014)

15. Hsu, C.-K. et al. Caveolin-1 controls hyperresponsiveness to mechanical stimuli and fibrogenesis-associated RUNX2 activation in keloid fibroblasts. J. Invest. Dermatol. 138, 208-218 (2018).

16. Ogawa, R. Mechanobiology of scarring. Wound Repair Regen. 19, S2-S9 (2011)

17. Aya, R. et al. The shear wave velocity on elastography correlates with the clinical symptoms and histopathological features of keloids. Plast. Reconstr. Surg. Glob. Open 3, e464 (2015).

18. Tachi, M. \& Iwamori, M. Mass spectrometric characterization of cholesterol esters and wax esters in epidermis of fetal, adult and keloidal human skin. Exp. Dermatol. 17, 318-323 (2008)

19. Calvo, F. et al. Mechanotransduction and YAP-dependent matrix remodelling is required for the generation and maintenance of cancer-associated fibroblasts. Nat. Cell Biol. 15, 637-646 (2013).

20. Liu, F. et al. Mechanosignaling through YAP and TAZ drives fibroblast activation and fibrosis. Am. J. Physiol. Lung Cell. Mol. Physiol. 308, L344-L357 (2015).

21. Sun, L.-P., Seemann, J., Goldstein, J. L. \& Brown, M. S. Sterol-regulated transport of SREBPs from endoplasmic reticulum to Golgi: Insig renders sorting signal in Scap inaccessible to COPII proteins. Proc. Natl Acad. Sci. USA 104, 6519-6526 (2007).

22. Watanabe, K. et al. A ROCK inhibitor permits survival of dissociated human embryonic stem cells. Nat. Biotechnol. 25, 681-686 (2007).

23. Ohgushi, M. et al. Molecular pathway and cell state responsible for dissociation-induced apoptosis in human pluripotent stem cells. Cell Stem Cell 7, 225-239 (2010).

24. Storey, M. K., Byers, D. M., Cook, H. W. \& Ridgway, N. D. Cholesterol regulates oxysterol binding protein (OSBP) phosphorylation and golgi localization in chinese hamster ovary cells: correlation with stimulation of sphingomyelin synthesis by 25 -hydroxycholesterol. Biochem. J. 336, 247-256 (1998).

25. Mohammadi, A. et al. Golgi localization and phosphorylation of oxysterol binding protein in Niemann-Pick C and U18666A-treated cells. J. Lipid Res. 42, 1062-1071 (2001).

26. De Matteis, M. A. \& Godi, A. Protein-lipid interactions in membrane trafficking at the Golgi complex. Biochim. Biophys. Acta 1666 264-274 (2004).

27. D'Souza-Schorey, C. \& Chavrier, P. ARF proteins: roles in membrane traffic and beyond. Nat. Rev. Mol. Cell Biol. 7, 347-358 (2006).

28. Walker, A. K. et al. A conserved SREBP-1/phosphatidylcholine feedback circuit regulates lipogenesis in metazoans. Cell 147, 840-852 (2011).

29. Smulan, L. J. et al. Cholesterol-independent SREBP-1 maturation is linked to arf1 inactivation. Cell Rep. 16, 9-18 (2016).

30. Takashima, K. et al. COPI-mediated retrieval of SCAP is crucial for regulating lipogenesis under basal and sterol-deficient conditions. J. Cell Sci. 128, 2805-2815 (2015).

31. Zhang, P. \& Reue, K. Lipin proteins and glycerolipid metabolism: roles at the ER membrane and beyond. Biochim. Biophys. Acta 1859, 1583-1595 (2017).

32. Peterson, T. R. et al. mTOR complex 1 regulates lipin 1 localization to control the srebp pathway. Cell 146, 408-420 (2011).

33. Fernández-Ulibarri, I. et al. Diacylglycerol is required for the formation of COPI vesicles in the Golgi-to-ER transport pathway. Mol. Biol. Cell 18, 3250-3263 (2007).

34. Asp, L. et al. Early stages of Golgi vesicle and tubule formation require diacylglycerol. Mol. Biol. Cell 20, 780-790 (2009).
35. Baron, C. L. \& Malhotra, V. Role of diacylglycerol in PKD recruitment to the TGN and protein transport to the plasma membrane. Science 295, 325-328 (2002).

36. Antonny, B., Huber, I., Paris, S., Chabre, M. \& Cassel, D. Activation of ADP-ribosylation factor 1 GTPase-activating protein by phosphatidylcholinederived diacylglycerols. J. Biol. Chem. 272, 30848-30851 (1997).

37. Harris, T. E. et al. Insulin controls subcellular localization and multisite phosphorylation of the phosphatidic acid phosphatase, lipin 1. J. Biol. Chem. 282, 277-286 (2007)

38. Péterfy, M., Harris, T. E., Fujita, N. \& Reue, K. Insulin-stimulated interaction with 14-3-3 promotes cytoplasmic localization of lipin-1 in adipocytes. J. Biol. Chem. 285, 3857-3864 (2010).

39. Hausser, A. et al. Protein kinase D regulates vesicular transport by phosphorylating and activating phosphatidylinositol-4 kinase III $\beta$ at the Golgi complex. Nat. Cell Biol. 7, 880-886 (2005)

40. Villani, M. et al. Sphingomyelin synthases regulate production of diacylglycerol at the Golgi. Biochem. J. 414, 31-41 (2008).

41. Liu, G.-H. \& Gerace, L. Sumoylation regulates nuclear localization of lipin-1 $\alpha$ in neuronal cells. PLoS ONE. 4, e7031 (2009).

42. Li, T. Y. et al. Tip60-mediated lipin 1 acetylation and ER translocation determine triacylglycerol synthesis rate. Nat. Commun. 9, 1916 (2018).

43. Isermann, P. \& Lammerding, J. Nuclear mechanics and mechanotransduction in health and disease. Curr. Biol. 23, R1113-R1121 (2013).

44. Coste, B. et al. Piezo1 and Piezo2 are essential components of distinct mechanically activated cation channels. Science 330, 55-60 (2010).

45. Egea, G., Lázaro-Diéguez, F. \& Vilella, M. Actin dynamics at the Golgi complex in mammalian cells. Curr. Opin. Cell Biol. 18, 168-178 (2006).

46. Gurel, P. S., Hatch, A. L. \& Higgs, H. N. Connecting the cytoskeleton to the endoplasmic reticulum and golgi. Curr. Biol. 24, R660-R672 (2014)

47. Guet, D. et al. Mechanical role of actin dynamics in the rheology of the Golgi complex and in Golgi-associated trafficking events. Curr. Biol. 24 1700-1711 (2014).

48. Fu, J. et al. Mechanical regulation of cell function with geometrically modulated elastomeric substrates. Nat. Methods 7, 733-736 (2010).

49. Sato, T. \& Clevers, H. Growing self-organizing mini-guts from a single intestinal stem cell: mechanism and applications. Science 340, 1190-1194 (2013)

50. McFarlane, M. R. et al. Scap is required for sterol synthesis and crypt growth in intestinal mucosa. J. Lipid Res. 56, 1560-1571 (2015).

51. Katsuda, T. et al. Conversion of terminally committed hepatocytes to culturable bipotent progenitor cells with regenerative capacity. Cell Stem Cell 20, 41-55 (2017).

52. Creutz, C. E., Eaton, J. M. \& Harris, T. E. Assembly of high molecular weight complexes of lipin on a supported lipid bilayer observed by atomic force microscopy. Biochemistry 52, 5092-5102 (2013).

\section{Acknowledgements}

The authors thank Y. Chen, K. Mori, G. DelSal, B. Viollet, H. Louvel, E. Greotti, A. DeMatteis, R. Venditti, A. Hausser, R. Rizzuto, D. Vecellio Reane, L. Scorrano, L. Pernas, C. Cheng, E. Melloni, M. Pende, R. Talha, R. Ogawa, J. Goldstein P. Espenshade and W. Shao for protocols and materials, S. Giulitti for generous help with hydrogels, M. Pellegrini and I. Zorzan for help with hPSC cultures and G. Martello and M. Montagner for thoughtful discussions. This work was supported by AIRC IG-15307 and IG-21392, WCR 15-1192, CARIPARO Eccellenza 2017 and University of Padua BIRD grants to S.D., an AIRC Hard ROCK Café Fellowship to G.S., the UPMC 'Interface pour le Vivant' doctoral programme to S.M. and an AIRC Special Program Molecular Clinical Oncology ‘ 5 per mille’ 10016 to S.B.

\section{Author contributions}

P.R. and I.B. performed experiments and analysed data with help from G.S. and A.P. M.A., S.P. and N.M. performed metabolic measurements and advised on the interpretation of metabolic data. S.M. and J.B.M. performed Golgi micromanipulations. M.F. and S.B. performed bioinformatics analyses. S.D. and P.R. planned experiments. S.D coordinated and supervised the project, and wrote the paper.

\section{Competing interests}

The authors declare no competing interests.

\section{Additional information}

Supplementary information is available for this paper at https://doi.org/10.1038/ s41556-018-0270-5.

Reprints and permissions information is available at www.nature.com/reprints. Correspondence and requests for materials should be addressed to S.D.

Publisher's note: Springer Nature remains neutral with regard to jurisdictional claims in published maps and institutional affiliations.

(c) The Author(s), under exclusive licence to Springer Nature Limited 2019 


\section{Methods}

Reagents. Plasmids encoding for SCAP-MYC, HA-S1P and HA-S2P were from Y. Chen (SIBS Shanghai), perilipin3-RFP from L. Scorrano (UniPd), kinasedead GFP-PKD1-K612W from A. Hausser (UniStuttgart), ARF1-T31N-GFP from A. DeMatteis (TIGEM Naples), KDEL-mCherry from E. Greotti (UniPd) and GFP-OSBP from N. Ridgway (UniGlasgow). Addgene plasmids: FASN-lux \#8890; LDLR-lux \#14940; 8XGTIIC-lux \#34615; NF2 \#19701; CMV-Luc2P_ARE \#62857; constitutive-active FLAG-SREBP2 \#26807; FLAG-Lipin-1 WT \#32005 and 17S/A \#32007; mCherry-Golgi(B4GALT1) \#55052. Full-length SREBP1 and SREBP2 were subcloned from Addgene plasmids \#32017 and \#32018. ER/Golgi membrane-tethered Lipin-1 isoforms were obtained by in-frame fusion of the Syntaxin 5 delta220 or TMD fragments $\mathrm{s}^{53,54}$ to FLAG-Lipin-1 WT and have been deposited as Addgene plasmids \#120277 and \#120278. SUMOylation (K616/646A) and acetylation (K476/646A) FLAG-Lipin-1 mutants were obtained by targeted mutagenesis. All plasmids were sequence-verified before use and transfected as endotoxin-free maxi preps.

The small molecule inhibitors were Y27632 (Axon 1683, $20 \mu \mathrm{M}, 10 \mu \mathrm{M}$ on hPSC), Fasudil/HA1077 (SantaCruzBiotechnology sc358231, $20 \mu$ M), ML7 (Sigma I2764, $20 \mu \mathrm{M}$ ), blebbistatin (Sigma B0560, $20 \mu \mathrm{M}$ ), propranolol (Sigma P0884, $100 \mu \mathrm{M}$ ), cycloheximide (Sigma C1988, $100 \mu \mathrm{g} \mathrm{ml}^{-1}$ ), TOFA (Sigma T6575, $15 \mu \mathrm{M}$, $75 \mathrm{nM}$ on hPSC), cerivastatin (Sigma SML0005, $10 \mu \mathrm{M}, 25 \mathrm{nM}$ on hPSC), 25-hydroxycholesterol (Sigma SML2042, 30 $\mu \mathrm{M}, 1.25 \mu \mathrm{M}$ on hPSC), U18666A (SantaCruzBioTechnology sc203306, $3 \mu \mathrm{M}$ ), PF429242 (Sigma SML0667, $10 \mu \mathrm{M}$ ), Z-VAD-FMK (Sigma V116, $30 \mu \mathrm{M}$ ), Torin1 (Axon 1833, $500 \mathrm{nM}$ ), MG132 (Sigma C2211, 10 $\mu$ M) and MG115 (Sigma C6706, $10 \mu \mathrm{M}$ ).

siRNAs were selected among FlexiTube GeneSolution 4 siRNA sets (Qiagen) and reordered after validation as dTdT-overhanging $19 \mathrm{nt}$ RNA duplexes (Thermo). siRNA sequences are provided in Supplementary Table 1. For AMPKa1/2 siRNA mix a and $b$ see ref. ${ }^{55}$

Cell cultures. MCF10A, MCF10ATk1 and MCF10ATk1 pBABE mTAZ 4SA were cultured in DMEM/F12 5\% HS insulin (Sigma), cholera toxin (Sigma), hEGF (Peprotech) and hydrocortisone (Sigma), GFP-Rab6 and parental RPE1 in DMEM/F12 10\% FBS (neomycin for GFP-Rab6), MDA-MB-231 in DMEM/ F12 10\% FBS, HEK293 in DMEM 10\% FBS, WI-38 in MEM 10\% FBS 5\% oxygen, 3T3-L1 in DMEM 10\% FBS 5\% oxygen, H9 hPSC in E8 medium (DMEM/F12, $\mathrm{NaHCO}_{3}$, insulin, selenium, transferrin, L-ascorbic acid, FGF2, TGFb1) $5 \%$ oxygen, and WT and AMPKa1 $/ 2^{--}$MEFs in DMEM $20 \%$ FBS. Glutamine was freshly added to a final concentration of $2 \mathrm{mM}$ to all media. General media, serum and supplements were from Thermo. All cell lines were routinely tested with an ATCC Universal Mycoplasma Detection kit to exclude contaminations. Cells for immunofluorescence in a stiff microenvironment were plated on fibronectincoated glass coverslips. Stiff $(E \approx 15 \mathrm{kPa})$ and soft $(E \approx 0.5 \mathrm{kPa})$ fibronectin-coated polyacrylamide hydrogels were assembled in-house by standard protocols. Annular-shaped micropatterns ( 25 or $35 \mu \mathrm{m}$ diameter, $5 \mu \mathrm{m}$ line thickness) were printed on PEG-coated glass coverslips by deep ultraviolet photolithography and coated with $50 \mathrm{\mu g} \mathrm{ml}^{-1}$ fibronectin supplemented with $20 \mu \mathrm{g} \mathrm{ml}^{-1}$ Alexa647fibrinogen (Sigma). hPSCs were dissociated with Tryple (Thermo) and plated as single cells (2,500 cells per $2 \mathrm{~cm}^{2}$ well) in the presence or absence of the indicated small molecules for $24 \mathrm{~h}$; medium was then changed and cells were left growing for 4-5 days, until the appearance of macroscopic colonies. Treatment of colonies was done on colonies grown from single cells as above, and then treating for $24 \mathrm{~h}$. Cell transfections were carried out with Transit-LT1 (MirusBio) or with Lipofectamine RNAi-MAX (Thermo)

Antibodies, immunofluorescence and stains. See Supplementary Table 2 for catalogue numbers, dilutions and validations. For the SREBP2 antibodies, independent lots were used with consistent results. Immunofluorescence was performed as in ref. ${ }^{56}$ with minor modifications: $10 \mathrm{~min} 1.5 \mathrm{mg} \mathrm{ml}^{-1}$ glycine in PBS before permeabilization to reduce background, and blocking in 1-2\% BSA Images were acquired sequentially with a Zeiss LSN700 or a Leica SP5 confocal microscope equipped with a charge-coupled device camera using ZEN 2 or Leica LAS AF software. Typical acquisition settings for IF were as follows: image size $1,024 \times 1,024$ pixels; acquisition mode $x y z$; pixel size $0.15 \mu \mathrm{m}$; image depth 8 bits; acquisition speed 5/10, with average 2; Plan-Apochromat $\times 63 / 1.40$ oil DIC M27 objective. For multichannel acquisitions, we used a main beamsplitter 405/488/555/639. Raw images (saved in .czi or .lif formats) were opened in ImageJ and saved in exportable formats. If needed, colours were changed (for example, red to green) with Photoshop CC

Oil Red-O staining (Sigma) was carried out after fixation, in $60 \% \mathrm{vol} / \mathrm{vol}$ isopropanol/water. C11-Bodipy581/591 (Thermo) was incubated for $15 \mathrm{~min}$ with cells before flow cytometry analysis. Filipin staining was acquired using an ultraviolet filter set $(340 / 380 \mathrm{~nm}$ excitation, $40 \mathrm{~nm}$ dichroic, $430 \mathrm{~nm}$ long pass filter) by prefocusing cells based on TOTO3 nuclear counterstain $(633 / 647 \mathrm{~nm})$ and then acquiring the ultravioletUV channel without prior observation to avoid photobleaching. Typical acquisition settings for Filipin were as follows: image size $1,024 \times 1,024$ pixels; acquisition mode $x y z$; pixel size $0.15 \mu \mathrm{m}$; image depth 8 bits; acquisition speed $5 / 10$, with average 2 ; Plan-Apochromat $\times 63 / 1.40$ oil DIC M27 objective. Pictures were always taken using the same acquisition conditions for all different experimental samples; panels are representative pictures based on at least two independent experiments. Quantifications and $n$ are provided in Supplementary Table 3.

Alkaline phosphatase staining was carried out with a Leukocyte AP kit (Sigma). Panels shown are representative pictures of one biological replicate of one experiment; each experiment was repeated three times independently. Quantifications and $n$ are provided in Supplementary Table 3.

\section{Cell fractionation, western blotting and pulldown. Nuclear extracts and} microsomes were obtained by resuspending cells in lysis buffer $(250 \mathrm{mM}$ sucrose, $10 \mathrm{mM}$ triethanolamine $\mathrm{pH} 7.4,10 \mathrm{mM}$ acetic acid, $1 \mathrm{mM} \mathrm{EDTA}, 10 \mathrm{mM} \mathrm{KCl}$ ) and passing cells eight times (that is, complete lysis at the microscope) through an Isobiotec Cell Homogenizer with a $6 \mu \mathrm{m}$ clearance sphere. Lysates were centrifuged at 800 r.c.f. to isolate nuclei, and then at 100,000 r.c.f. to isolate microsomes. Western blotting was performed as in ref. ${ }^{57}$; in Fig. $3 \mathrm{e}$ and Supplementary Fig. $3 \mathrm{~h}$ cells were incubated with $10 \mu \mathrm{M}$ MG132/MG115 proteasome inhibitors during treatment to prevent degradation of cleaved SREBPs. Active GTP-bound ARF1 pulldown was performed with a commercial kit following the manifacturer's intructions (Cytoskeleton BK032).

Gene expression studies. Luciferase assays were performed in MDA-MB-231 cells as in ref. ${ }^{55}$. For qPCR, total RNA was isolated using commercial kits with DNAse treatment (Qiagen, Norgen). cDNA synthesis was carried out with M-MLV Reverse Transcriptase (Thermo) and oligo-dT primers. qPCR reactions were assembled with FastStart SYBR Green Master Mix (Roche) and run on a QuantStudio6 thermal cycler (Thermo). Gene expression levels for each biological sample were quantified as the mean between three technical replicates; GAPDH expression levels were used to normalize gene expression between samples, based on eff ${ }_{\text {gene }}^{-\Delta \text { gene }_{\text {te }}} / \mathrm{eff}_{\mathrm{GAPDH}}^{-\Delta C t_{\mathrm{GAPH}}}$ where eff is the primer amplification efficiency, calculated from three consecutive fivefold dilutions of one control sample cDNA, centred on the dilution used for all other samples. Primer sequences are provided in Supplementary Table 1.

Microarray probe synthesis, hybridization and detection were performed at CMB Trieste on HumanHT-12 v4 Expression BeadChips with an Illumina Hiscan system. Data analyses were performed in R (version 3.0.2) using Bioconductor libraries (BioC 2.13) and R statistical packages. Probe level signals were converted to expression values using the Robust Multi-array Average procedure RMA ${ }^{58}$ of the Bioconductor Affymetrix package. Differentially expressed genes were identified using the Significance Analysis of Microarray (SAM) algorithm coded in the same $R$ package ${ }^{59}$. In SAM, we estimated the percentage of false-positive predictions (that is, false discovery rate, FDR) with 100 permutations. Genes activated or inhibited upon YM treatment and used for gene list enrichment analysis were filtered based on $P<0.05$ and fold change $>1.3$. Gene list enrichment analysis was performed with Enrichr ${ }^{60}$. SREBP target genes were defined based on refs. ${ }^{61,62}$.

Metabolomics and metabolic analyses. Large-scale metabolic analysis (global metabolomics) and principal component analysis of the results were carried out by Metabolon. Mechanosensitive MCF10Tk1 cells ${ }^{56}$ were washed once in warm $1 \times$ PBS, and metabolites were extracted for $5 \mathrm{~min}$ at room temperature on $15 \mathrm{~cm}$ plates with $80 \% \mathrm{vol} / \mathrm{vol}$ methanol/water extraction buffer with internal standards. We harvested cells on plate to specifically avoid alteration of actin tension and metabolism due to cell detachment. Metabolites were normalized to protein content. This analysis did not focus on triglyceride content. Clustering of selected lipid metabolites (fold change $>2.5, P<0.05$ ) shown in Supplementary Fig. 1e was carried out with Heatmapper.

Targeted lipidomic analysis was carried out on MCF10Tk1 cells lysed in 1:1 vol/ vol MeOH/acetonitrile extraction buffer by tissue lyser and spun at 20,000 $\mathrm{g}$ for $5 \mathrm{~min}$ at $4^{\circ} \mathrm{C}$. Supernatants were then passed through a regenerated cellulose filter, dried, and resuspended in $100 \mu \mathrm{l} \mathrm{MeOH}$.

For quantification of the different phospholipid species, liquid chromatography tandem mass spectrometry LC-MS/MS analysis was performed on an API4000 triple quadrupole mass spectrometer (AB Sciex) coupled with an HPLC system (Agilent) and CTC PAL HTS autosampler (PAL System). The identity of the different phospholipid families was confirmed using pure standards, namely one for each family. Methanolic extracts were analysed with a $5 \mathrm{~min}$ run in both positive and negative ion mode with 275 multiple reaction monitoring (MRM) transitions in positive mode and 92 MRM transitions in negative mode. Quantification of different phospholipids in positive ion mode was performed using a Synergi $4 \mu$ Hydro-RP $(50 \mathrm{~mm} \times 2.0 \mathrm{~mm}, 4 \mu \mathrm{m}$; Phenomenex $)$ and in negative ion mode using a Cyano-phase LUNA column $(50 \mathrm{~mm} \times 4.6 \mathrm{~mm}, 5 \mu \mathrm{m}$; Phenomenex). The mobile phase for positive ion mode was $0.1 \%$ formic acid in $\mathrm{MeOH}$ and $5 \mathrm{mM}$ ammonium acetate $\mathrm{pH} 7 \mathrm{in} \mathrm{MeOH}$ for negative ion mode, both with a flow rate of $500 \mu \mathrm{min}^{-1}$. MultiQuant software version 3.0.2 was used for data analysis and peak review of chromatograms. Semi-quantitative evaluation of different phospholipids was performed based on external standards, then the data were normalized on protein content assessed by the BCA (bicinchoninic acid assay) method.

For quantification of the different diacylglycerol and triacylglycerol species the LC-MS/MS analysis was performed on an API-4000 triple quadrupole mass 
spectrometer (AB Sciex) coupled with an HPLC system (Agilent) and a CTC PAL HTS autosampler (PAL System). Methanolic extracts were dried under nitrogen and resuspended in $100 \mu \mathrm{l}$ of $65 \%$ acetonitrile/30\% isopropanol/5\% water. Samples were then analysed with a 10 min run in positive ion mode with $92 \mathrm{MRM}$ transitions. Quantification of different diacylglycerols and triacylglycerols was performed using an XBridge C-18 column ( $100 \mathrm{~mm} \times 2.1 \mathrm{~mm}, 3.5 \mu \mathrm{m}$; Waters). Column temperature was set at $55^{\circ} \mathrm{C}$. The mobile phases were as follows-phase A: $40 \%$ acetonitrile, $0.1 \%$ formic acid and $10 \mathrm{mM}$ ammonium acetate in water; phase B: isopropanol $90 \%$, acetonitrile $10 \%, 0.1 \%$ formic acid and $10 \mathrm{mM}$ ammonium acetate; $T_{0}: 55 \% \mathrm{~A}$; $T_{2 \min }: 55 \% \mathrm{~A} ; T_{4 \min }: 3 \% \mathrm{~A} ; T_{7 \min }: 3 \% \mathrm{~A} ; T_{7.1 \mathrm{~min}}: 55 \% \mathrm{~A} ; T_{10 \min }: 55 \% \mathrm{~A}$; with a flow rate of $260 \mu \mathrm{lmin}^{-1}$. MultiQuant software version 3.0.2 was used for data analysis and peak review of chromatograms. Semi-quantitative evaluation of different diacylglycerols and triacylglycerols was performed based on external standards, then data were normalized on protein content assessed by the BCA method.

For isotopologue analysis, cells were exposed to $2.5 \mathrm{mM}\left[{ }^{13} \mathrm{C}_{2}\right]$ acetate (Sigma 282014 ) for $24 \mathrm{~h}$. After removing medium, cells were washed in ice-cold PBS. Lipid extraction was performed by adding $500 \mu \mathrm{l}$ of MeOH/ACN $(1: 1 \mathrm{vol} / \mathrm{vol})$ and then $2.5 \mathrm{ml}$ of chloroform-MeOH (1:1 vol/vol). Total fatty acids were obtained by acid hydrolysis by adding $1.25 \mathrm{ml}$ of $\mathrm{HCl} 1 \mathrm{M}$ and $1.25 \mathrm{ml}$ of $\mathrm{MeOH}$. After leaving samples for $1 \mathrm{~h}$ under agitation at 210 r.p.m., $2.5 \mathrm{ml}$ of chloroform-water (1:1 vol/ vol) were added to the mixture and the lower organic phase was collected, split, transferred into tubes and dried under nitrogen flow. The residue was resuspended in $\mathrm{MeOH} / \mathrm{H}_{2} \mathrm{O}(1: 1 \mathrm{vol} / \mathrm{vol})$ and used for total fatty acid analysis. Samples were analysed with an API 4000 mass spectrometer (AB Sciex) coupled with an HPLC system (Agilent) and CTC PAL HTS autosampler (PAL System). The gradient (flow rate, $0.5 \mathrm{ml} \mathrm{min}^{-1}$ ) was as follows: $T_{0}: 20 \% \mathrm{~A} ; T_{20}: 1 \% \mathrm{~A} ; T_{25}: 1 \% \mathrm{~A} ; T_{251}: 20 \% \mathrm{~A}$; $T_{30}: 20 \% \mathrm{~A}$, where $\mathrm{A}$ is acetic acid $(15 \mathrm{mM})$ and $N$-ethylisopropylamine $(10 \mathrm{mM})$ in $\mathrm{H}_{2} \mathrm{O}: \mathrm{MeOH}$ 97:3 and B:MeOH. The Hypersil GOLD column (C8 $100 \mathrm{~mm} \times 3 \mathrm{~mm}$, $3 \mu \mathrm{m}$; Thermo-Scientific) was maintained at $40^{\circ} \mathrm{C}$ for all analyses.

For the quantification of free and total fatty acids and cholesterol levels, we used an aliquot of extracts as described above and commercial kits (Sigma MAK044 and MAK043), following the manufacturer's instructions.

Intracellular optical micromanipulation, microrheological measurements and analysis. The set-up combining optical trapping and confocal imaging has been described previously ${ }^{47}$. Briefly, red fluorescent $580 / 605 \mathrm{~nm} 2$ - $\mu \mathrm{m}$-diameter latex beads (Thermo F88265) were endocytosed overnight in RPE1 cells stably expressing the Golgi marker GFP-Rab6. The incubation time and bead concentration were adjusted so that cells typically contained one or two beads before optical micromanipulation. Cells were plated on 18 -mm-diameter coverslips uniformly coated with fibronectin, or with annular-shaped adhesive fluorescent micropatterns of different diameters $(25$ or $35 \mu \mathrm{m})$, for $6 \mathrm{~h}$. Non-adherent cells were washed off by rinsing with culture medium. The coverslip was then mounted in a Ludin chamber and the culture medium was supplemented with $20 \mathrm{mM}$ HEPES before the experiment.

Force was applied on GFP-positive Golgi membranes by first trapping a bead located close to the Golgi apparatus and then displacing the microscope stage to push the organelle against the trapped bead. Trapping was not possible on polyacrylamide hydrogels because of the excessive distance between the lens and cells in this set-up. The applied force $F$ was deduced from the bead displacement relative to the trap centre, $\Delta x$, after calibration of the trap stiffness

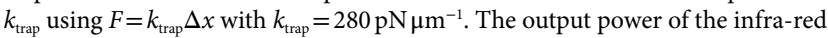
laser at the objective aperture was $150 \mathrm{~mW}$. Stage displacement was performed using a nanopositioning piezo-stage (Nanobio 200, Mad City Labs) controlled by NanoRoute3D software (Mad City Labs). The stage displacement consisted of five consecutive $0.5 \mu \mathrm{m}$ steps with a $10 \mathrm{~s}$ pause between each step to allow viscoelastic relaxation of the bead position towards the trap centre. The total duration of optical trapping was limited to $1 \mathrm{~min}$ for a given cell to ensure cell viability.

To characterize the rigidity of the microenvironment surrounding the bead, we used a phenomenological analysis of the relaxation curves as in refs. ${ }^{47,63}$ to measure three parameters: the frequency of bead ejection, the bead step amplitude and the rigidity index. Qualitatively, in a low rigidity microenvironment, friction on the bead is low and the bead does not move much from the trap centre during the step displacement and relaxes rapidly towards the trap centre. In a rigid microenvironment, the bead experiences a high friction and its initial displacement is larger and closer to the step displacement $(0.5 \mu \mathrm{m})$ and the relaxation is slower. If the force acting on the bead is too large (typically above $300-400 \mathrm{pN}$ ), the bead falls off the trap and subsequently follows the displacement of the stage. We termed such events 'ejections' and scored their frequency (defined as the ratio between the number of experiments in which ejection occurred and the total number of experiments) and the step at which ejection occurred. The bead step amplitude $X_{\mathrm{b}}$ corresponds to the displacement of the bead after a $0.5 \mu \mathrm{m}$ step of the piezo stage. Values of $X_{\mathrm{b}}$ close to $0.5 \mu \mathrm{m}$ indicate a high rigidity of the bead microenvironment. Lower values indicate softer microenvironments. The rigidity index (RI) is defined as

$$
\mathrm{RI}=\frac{\int_{t_{i}}^{t_{i}+T} x_{\mathrm{b}}(t) \mathrm{d} t}{\int_{t_{i}}^{t_{i}+T} x_{\mathrm{s}}(t) \mathrm{d} t}=\frac{\int_{t_{i}}^{t_{i}+T} x_{\mathrm{b}}(t) \mathrm{d} t}{X_{\mathrm{s}} T}
$$

where $t_{i}$ is the time when the $i$ th $0.5 \mu \mathrm{m}$ step displacement of the piezo stage occurs, $x_{\mathrm{b}}$ and $x_{\mathrm{s}}$ are, respectively, the displacement of the bead relative to the trap centre and the displacement of the piezo stage, $X_{\mathrm{s}}=0.5 \mu \mathrm{m}$ is the amplitude of the piezo-stage step and $T=10 \mathrm{~s}$ is the duration of the step. The rigidity index is a phenomenological parameter that allows us to compare the rigidity of the microenvironment surrounding the bead in various conditions. The value of rigidity index falls between 0 (the microenvironment does not exert any friction on the bead) and 1 (the microenvironment is not deformable). The values of the rigidity index for each step displacement were averaged.

To measure GFP-PKD-KD recruitment on force application, cells were plated at day 1 in a 12 -well plate to reach around $75 \%$ confluence on day 2 . At day 2 , cells were transfected with GFP-PKD-KD and mCherry-Golgi (B4GALT1) plasmids. At the end of day 2 , the cells were incubated with $2-\mu \mathrm{m}$-diameter fluorescent beads overnight. At day 3, cells were transferred to fibronectin-coated coverslips for the experiment. The evolution of normalized intensity of GFP-PKD-KD in the region of the Golgi apparatus, visualized by the mCherry-Golgi marker, was monitored after application of a mechanical constraint exerted by internalized beads trapped with optical tweezers. A bead located near the Golgi apparatus was selected in a cell expressing GFP-PKD-KD and the mCherry-Golgi marker. A first image was taken at $t=0 \mathrm{~min}$. The bead was then trapped with the optical tweezers and the microscope stage manually displaced to bring the Golgi apparatus in contact with the bead and apply a compressive constraint on the Golgi apparatus during $1 \mathrm{~min}$. The same protocol was repeated every $5 \mathrm{~min}$ until $t=30 \mathrm{~min}$. The duration of the compressive constraint was reduced to $30 \mathrm{~s}$ after $t=10 \mathrm{~min}$ to avoid cellular damage due to prolonged laser exposure. As a control, the same protocol was used but the microscope stage was displaced to move the bead away from the Golgi apparatus.

To quantify the fluorescence intensity of GFP-PKD-KD in each of the seven images taken every $5 \mathrm{~min}$, the Golgi apparatus was delimited using the mCherry Golgi marker. The total intensities of the mCherry Golgi marker $\left(I_{\mathrm{r}}\right)$ and GFPPKD-Kd $\left(I_{\mathrm{g}}\right)$ were measured as well as the mean intensity of the background for each channel ( $\left\langle I_{\mathrm{r} \text { back }}\right\rangle$ for the mCherry Golgi marker; $\left\langle I_{\mathrm{g} \text { back }}\right\rangle$ for GFP-PKD-KD) and the area of the Golgi apparatus, $A_{\text {Golgi }}$. The total intensity of the background in the Golgi apparatus region for each channel was then estimated by multiplying the mean background intensity by the Golgi area:

$$
\begin{aligned}
& I_{\text {r back }}=A_{\text {Golgi }}\left\langle I_{\text {r back }}\right\rangle \\
& I_{\text {g back }}=A_{\text {Golgi }}\left\langle I_{\text {g back }}\right\rangle
\end{aligned}
$$

The fluorescence intensity of GFP-PKD-KD was normalized by the fluorescence intensity of the mCherry Golgi marker:

$$
I=\frac{I_{\mathrm{g}}-I_{\mathrm{g} \text { back }}}{I_{\mathrm{r}}-I_{\mathrm{r} \text { back }}}
$$

to account for slight changes in the imaging plane from one image to the next. The relative temporal variations of the GFP-PKD-KD fluorescence were obtained by normalizing the intensity $I$ measured from each image taken every $5 \mathrm{~min}$ by its initial value $I_{0}: I(t)=I / I_{0}$.

Statistics and reproducibility. All data are based on two or three independent experiments with independent biological replicates, except for metabolomics and microarrays, which were based on independent biological replicates collected in a single experiment. Experimental repetitions were carried out by thawing a new aliquot of cells derived from the original stock. Key data were independently replicated by two different operators, across different cell lines, and with independent techniques providing coherent results. Data are presented as mean and single points, or mean and standard error of the mean (s.e.m.) as indicated in the figure legends. All $n$ values are pooled between independent experiments. Significance tests were unpaired non-parametric Mann-Whitney tests, unpaired two-tailed Student's $t$-tests, multiple unpaired two-tailed Student's $t$-tests with Holm-Sidak correction (for analysis of multiple qPCR markers) or Welch's two-sample $t$-tests (for global metabolomics). The $t$-tests were performed under the reasonable assumption that values follow a normal distribution and have similar variance.

Reporting summary. Further information on experimental design is available in the Nature Research Reporting Summary linked to this article.

\section{Code availability}

No custom codes were used in this study. All codes are indicated in the appropriate Methods sections and references.

\section{Data availability}

Microarray data generated here have been deposited in GEO under accession code GSE107275. Metabolomics and targeted lipidomics data have been deposited in the Figshare database (https://doi.org/10.6084/m9.figshare.7338764). The previously 
generated gene expression data analysed for Fig. $3 \mathrm{i}$ can be found in GEO under accession code GSE90051 (secondary data set). Source data for Figs. 1-7 and Supplementary Figs. 1-7 are provided in Supplementary Table 3. All other data supporting the findings of this study are available from the corresponding author on reasonable request.

\section{References}

53. Watson, R. T. \& Pessin, J. E. Transmembrane domain length determines intracellular membrane compartment localization of syntaxins 3,4 , and 5 . Am. J. Physiol. Cell Physiol. 281, C215-C223 (2001).

54. Misumi, Y., Sohda, M., Tashiro, A., Sato, H. \& Ikehara, Y. An essential cytoplasmic domain for the Golgi localization of coiled-coil proteins with a COOH-terminal membrane anchor. J. Biol. Chem. 276, 6867-6873 (2001).

55. Enzo, E. et al. Aerobic glycolysis tunes YAP/TAZ transcriptional activity. EMBO J. 34, 1349-1370 (2015)

56. Aragona, M. et al. A mechanical checkpoint controls multicellular growth through YAP/TAZ regulation by actin-processing factors. Cell 154, 1047-1059 (2013).
57. Santinon, G. et al. dNTP metabolism links mechanical cues and YAP/TAZ to cell growth and oncogene-induced senescence. EMBO J. 37, e97780 (2018).

58. Irizarry, R. A. et al. Exploration, normalization, and summaries of high density oligonucleotide array probe level data. Biostatistics 4, 249-264 (2003).

59. Tusher, V. G., Tibshirani, R. \& Chu, G. Significance analysis of microarrays applied to the ionizing radiation response. Proc. Natl Acad. Sci. USA 98, 5116-5121 (2001).

60. Kuleshov, M. V. et al. Enrichr: a comprehensive gene set enrichment analysis web server 2016 update. Nucleic Acids Res. 44, W90-W97 (2016).

61. Horton, J. D. et al. Combined analysis of oligonucleotide microarray data from transgenic and knockout mice identifies direct SREBP target genes. Proc. Natl Acad. Sci. USA 100, 12027-12032 (2003).

62. Porstmann, T. et al. PKB/Akt induces transcription of enzymes involved in cholesterol and fatty acid biosynthesis via activation of SREBP. Oncogene $\mathbf{2 4}$, 6465-6481 (2005).

63. Mandal, K., Asnacios, A., Goud, B. \& Manneville, J.-B. Mapping intracellular mechanics on micropatterned substrates. Proc. Natl Acad. Sci. USA 113, E7159-E7168 (2016). 


\section{Reporting Summary}

Nature Research wishes to improve the reproducibility of the work that we publish. This form provides structure for consistency and transparency in reporting. For further information on Nature Research policies, see Authors \& Referees and the Editorial Policy Checklist.

\section{Statistical parameters}

When statistical analyses are reported, confirm that the following items are present in the relevant location (e.g. figure legend, table legend, main text, or Methods section).
n/a Confirmed
$\square \bigotimes$ The exact sample size $(n)$ for each experimental group/condition, given as a discrete number and unit of measurement
$\square$ \An indication of whether measurements were taken from distinct samples or whether the same sample was measured repeatedly
$\square$ The statistical test(s) used AND whether they are one- or two-sided
$\square$ Only common tests should be described solely by name; describe more complex techniques in the Methods section.
Х $\square$ A description of all covariates tested
$\square$ \ A description of any assumptions or corrections, such as tests of normality and adjustment for multiple comparisons
$\square \bigotimes \begin{aligned} & \text { A full description of the statistics including central tendency (e.g. means) or other basic estimates (e.g. regression coefficient) AND } \\ & \text { variation (e.g. standard deviation) or associated estimates of uncertainty (e.g. confidence intervals) }\end{aligned}$
$\varnothing$ For null hypothesis testing, the test statistic (e.g. $F, t, r$ ) with confidence intervals, effect sizes, degrees of freedom and $P$ value noted
Х Give P values as exact values whenever suitable.
Х $\square$ For Bayesian analysis, information on the choice of priors and Markov chain Monte Carlo settings
Х $\square$ For hierarchical and complex designs, identification of the appropriate level for tests and full reporting of outcomes
Х $\square$ Estimates of effect sizes (e.g. Cohen's $d$, Pearson's $r$ ), indicating how they were calculated
$\varnothing$ Clearly defined error bars
State explicitly what error bars represent (e.g. SD, SE, CI)

Our web collection on statistics for biologists may be useful.

\section{Software and code}

Policy information about availability of computer code

Data collection $\quad$ RT-PCR data were acquired with QuantStudio 6\&7 Flex Software 1.0. Confocal images were acquired with Leica LAS AF or ZEN 2 softwares. Microarrays with Illumina Hiscan proprietary software. Western blotting was acquired with LAS400 ImageQuant 1.2. Stage displacements in Fig. 7 were controlled with NanoRoute3D software from Mad City Labs. MultiQuant ${ }^{\text {TM }}$ software version 3.0.2 was used for data analysis and peak review of chromatograms.

Data analysis

Data were analyzed with Microsoft Excel 15.32 or Prism 7. Microarray data were treated in R (version 3.0.2) using Bioconductor libraries (BioC 2.13) and R statistical packages referenced in the Online Methods and freely available. Gene list enrichment analysis was performed with Enrichr (http://amp.pharm.mssm.edu/Enrichr/). Heatmaps with Heatmapper (http://www.heatmapper.ca/).

MultiQuant ${ }^{\mathrm{TM}}$ software version 3.0.2 was used for metabolite data analysis and peak review of chromatograms. Images were treated

(crop, overlay, colors) with ImageJ or Adobe Photoshop according to NPG's image integrity rules. 
Policy information about availability of data

All manuscripts must include a data availability statement. This statement should provide the following information, where applicable:

- Accession codes, unique identifiers, or web links for publicly available datasets

- A list of figures that have associated raw data

- A description of any restrictions on data availability

Microarray data have been deposited (GEO database GSE107275). Metabolomics and targeted lipidomics data have been deposited (Figshare database 10.6084/ m9.figshare.7338764). Source data for Figs. 1-7 and Supplementary Figs. 1-7 have been provided in Supplementary Table 2. All other data supporting the findings of this study are available from the corresponding author on reasonable request.

\section{Field-specific reporting}

Please select the best fit for your research. If you are not sure, read the appropriate sections before making your selection.

$\bigotimes$ Life sciences $\quad \square$ Behavioural \& social sciences $\square$ Ecological, evolutionary \& environmental sciences

For a reference copy of the document with all sections, see nature.com/authors/policies/ReportingSummary-flat.pdf

\section{Life sciences study design}

All studies must disclose on these points even when the disclosure is negative.

Sample size

Sample size calculation was not performed. Sample size was chosen based on standards in the field and is indicated in figure legends and in Supplementary Table 2, which reports all source data for each dataset in this study. All qPCR experiments have been repeated at least two times independently, with at least two biological replicates each time, and each biological replicate was quantified by using 3 technical replicates (which were excluded from statistical analyses). All luciferase experiments have been repeated at least two times independently, with at least two biological replicates each time. All immunoblots, immunofluorescences and stains have been repeated at least two times independently.

Data exclusions No data were excluded including negative results.

Replication All data are the result of independently-repeated experiments with independent biological samples. All experimental repetitions were carried out by thawing a new aliquot of cells deriving from the original stock. Several key data were replicated across different cell lines, and with independent techniques (metabolomics-direct lipid quantification-lipid stains; microarrays-qPCR-luciferase assays; immunofluorescence with two independent anti-SREBP2 antibodies; SREBP1/2 inhibition by siRNA knockdown, 25OHC supplementation, S1P-inhibitor treatment; Lipin1 inhibition by propranolol treatment and by siRNA knockdown; AMPK inhibition by siRNA knockdown and knockout). Key data were independently replicated by two different operators. All attempts at replication were successful, provided that internal controls for technical reliability were acceptable.

Randomization Randomization was not relevant. Samples were allocated to experimental groups based on the treatments/experimental conditions applied, and their identity was known during experimentation and data analysis.

\begin{tabular}{l|l} 
Blinding & Analysis of metabolites were blinded, and hierarchical clustering indicated significant differences and correct grouping before sample identity
\end{tabular} disclosure. Immunofluorescences and stains were acquired by randomly selecting cells based on nuclear counterstain. All other analyses were not blinded because this was not applicable.

\section{Reporting for specific materials, systems and methods}

Materials \& experimental systems

Involved in the study
$\square$ Unique biological materials
$\square$ Antibodies
$\square$ Eukaryotic cell lines
$\square$ Palaeontology
$\square$ Human research participants

\begin{tabular}{l|l}
\multicolumn{2}{l}{ Methods } \\
\hline n/a Involved in the study \\
$\searrow$ ChIP-seq \\
$\searrow$ \\
$\square$ Flow cytometry \\
$\square$ MRI-based neuroimaging
\end{tabular}


Policy information about availability of materials

Obtaining unique materials Unique plasmids produced in this work (ER/Golgi membrane-bound FLAG-tagged Lipin-1 expression plasmids) are under submission to Addgene as Plasmids \#120277 and \#120278

\section{Antibodies}

Antibodies used

See Supplementary Table 1

Validation

See Supplementary Table 1

\section{Eukaryotic cell lines}

Policy information about cell lines

Cell line source(s)

MCF10ATk1. S. Santner.

MCF10ATk1 pBABE and pBABE mTAZ 4SA. Aragona et al., Cell 2013.

MCF10A. J. Brugge.

Parental and GFP-Rab6 RPE1. B. Goud (ATCC).

MDA-MB-231. ATCC.

HEK293. ATCC.

WI-38. ECACC.

3T3-L1. E. Melloni.

H9 hPSC. G. Martello.

WT and AMPKa1/2-/- MEFs. B. Viollet and H. Louvel.

Authentication

MCF10ATK1. These cells form tumors in the mammary fat pad of SCID mice and form filled spheres in 3D matrigel, at difference with MCF10A.

MCF10ATK1 pBABE mTAZ 4SA. These cells are positive for FLAG-tagged TAZ by WB, carry the expected antibiotic resistance, and scatter in 3D matrigel, at difference with parental MCF10ATK1.

MCF10A. These cells do not form tumors in the mammary fat pad of SCID mice and form hollow spheres in 3D matrigel, at difference with MCF10ATk1.

GFP-Rab6 RPE1. These cells are positive for Rab6-GFP by IF and carry the expected antibiotic resistance.

MDA-MB-231. ATCC.

HEK293. ATCC

WI-38. These cells undergo senescence upon expression or H-Ras (Santinon et al., EMBO J 2018).

3T3-L1. These cells differentiate into adipocytes upon treatment with the adequate differentiation induction/maintenance media.

H9 hPSC. These cells maintain pluripotency markers and clonogenic ability unless challenged with differentiating conditions. WT and AMPKa1/2-/- MEFs. Western blotting for AMPKa was used as validation.

Mycoplasma contamination

All cell lines were routinely tested negative for mycoplasma contamination, as stated in the appropriate Methods section.

Commonly misidentified lines

(See ICLAC register)

No commonly misidentified cell lines were used in the study. 